\title{
Whole-Liver Decellularization: Advances and Insights into Current Understanding
}

\author{
Ibrahim Fathi and Ahmed Eltawila
}

Additional information is available at the end of the chapter

http://dx.doi.org/10.5772/intechopen.69487

\begin{abstract}
Whole-liver decellularization comprises the removal of the antigenic cellular content, while maintaining intact 3D extracellular matrix architecture and the complex native vascular cues. As a result, it challenges the classical hurdles of xenotransplantation and hypothetically allows the production of bioengineered human-size liver constructs. The associated technique and understanding of the determinants of successful application evolved rapidly during the last decade. In this chapter, the authors offer a comprehensive walk-through, starting from the simplicity of the concept to the complexity of clinical application. Avoiding repetition, the chapter covers the fundamentals and advances of decellularization, recellularization, ex vivo perfusion culture, and sterilization techniques. The interplay between the main pivots of whole-liver decellularization, namely intrinsic matrix potentials, immune response, and vasculature is described. An effort was made to dissect the hurdles facing the whole-liver decellularization approach and to highlight the gaps in current literature. The authors also offer insights about some critical concepts including intra-scaffold flow dynamics, gradient zonation, critical cell mass/density, mechano-sensitivity, substrate modifications, nondestructive analysis, and the surgeon's perspective, together with the discussion of published in vivo trials and large-scale production parameters.
\end{abstract}

Keywords: liver, decellularization, recellularization, xenograft, mechano-sensitivity, flow dynamics, sterilization, thrombosis, nondestructive analysis, substrate modification

\section{Introduction}

The whole-liver decellularization (WLD) approach, one of the organ-engineering and xenotransplantation approaches, comprises the removal of hepatic cellular content through perfusion decellularization while maintaining an intact 3D extracellular matrix (ECM) scaffold and the native hepatic vascular network. Intact vasculature can then be used to 
repopulate the scaffold (a process termed "recellularization"), and thereafter allow the delivery of nutrients/oxygen to the newly seeded cells in a bioreactor setting, and in vivo after transplantation. Thus, a functional human-scale bioengineered liver can be fabricated, which is devoid of the original cellular antigenic content, repopulated with allogeneic or patient-specific cells, and relies on the ECM potentials for supporting cell proliferation and differentiation.

The main target of WLD approach is to provide physiologically matching liver grafts of xenogeneic origin for clinical transplantation and thus significantly expanding the organ harvest pool, which is the main focus herein. However, several other possible applications exist including drug testing, production of hydrogels and flask-coating materials, incorporation into ex vivo liver-support devices, or the use in repair of other tissues in the form of membranes (Figure 1). In this chapter, the authors offer a comprehensive walk-through of the different aspects of WLD, with the aim of highlighting the inadequacies and advances, clarifying the gaps in the approach hierarchy, and offering possible explanations and few theoretical insights. The main focus is to discuss and link the findings of the previous relevant studies, rather than inclusively listing them.

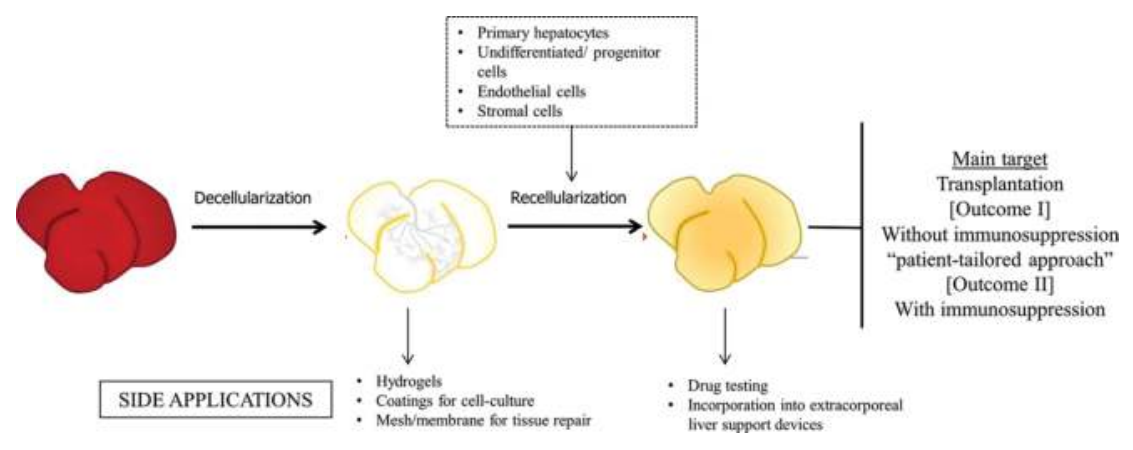

Figure 1. Schematic presentation of the whole-liver decellularization applications.

\section{Concept}

The concept of tissue decellularization was designed to evade the classical barriers to xenotransplantation and, thus, offers enormous enlargement of the tissue and organ pool for tissue repair and transplantation, as provoked by donor/patient mismatch crisis. The concept strongly relies on the intrinsic potentials of ECM as its fundamental justification. The whole-organ decellularization strategy was a step forward after clinical and experimental success of decellularized biological membranes by applying the same principles to whole organs in order to supply physiologicalsize-matched scaffolds for organ engineering. The cornerstone of this advance is the reliance on the native organ vascular system as a cue for perfusion decellularization, recellularization, and 
nutrients/oxygen delivery after in vivo transplantation. As a result, an expansion past the limitations of diffusion distance for non-vascularized grafts (about $200 \mu \mathrm{m}$ ) can be achieved. The decellularized whole-liver matrix (wDLM) therefore can hypothetically evade the xenograft immune rejection cascade by the removal of cellular antigens, supply physiological-size-matched constructs through reliance on the native vascular system, and allow for acellular scaffold sterilization, thus diminishing the risk of xenozoonosis.

In fact, the attractive potentials of ECM provoked the design of different approaches to produce ECM scaffolds that can be used for cell seeding or tissue repair, and offered as an on-shelf product. These approaches can be categorized into synthetic and natural ECM scaffold production techniques. Although great advancements were achieved in the ECM synthetic techniques, the complexity of native organ ECM and vasculature hinders the progress into organ constructs through this pathway. Natural ECM scaffolds, on the other hand, can be produced by either in vitro cell culture and further decellularization or borrowing the native tissue/organ ECM through tissue decellularization techniques. The complex liver architecture and vasculature renders the WLD the most attractive approach for liver ECM scaffold production on a logical scale.

Among other xenotransplantation approaches, the simplicity of the WLD approach also acquires it a relative advantage. As opposed to genetic manipulation approaches for instance, WLD rather represents a simple combination of natural ECM potentials and human cell populations, and therefore involves borrowing of functional elements rather than modifying them to fit the human physiology. It simply aims to shift xenotransplants to the allotransplants or auto-transplants zone (if patient-specific cells are used). Genetic modification on the other hand requires more complex techniques and detailed appreciation of all the significant differences in antigenic expression and cell function effectors. However, despite the clear hypothetical simplicity, the application trials repeatedly demanded for deeper understanding and robust refinement of the decellularization/recellularization techniques provoked by suboptimal functions and ultimately the short-term in vivo survival after transplantation.

In order to simplify the hierarchy of WLD, three main parameters that largely govern its success are identified, namely sufficient DLM intrinsic potentials, avoidance of adverse immune/host responses, and maintenance of patent hepatic vasculature. Other parameters including cell sources and sterilization for example can then be considered as cofactors and not integral in the proof of concept paradigm. The impact of insufficiencies related to these three basic elements can, however, overlap in various study designs. Therefore, the exact delineation of error becomes a difficult task. For example, both insufficiencies related to the vascular system and scaffold immunogenicity can result in vascular thrombosis. Also, poor intrinsic ECM properties can result in endothelialization defects and therefore vascular incompetency. Therefore, in order to accurately locate and troubleshoot the problems along the WLD process, isolated testing for each of the three parameters is necessary as distinct main categories, followed by their combination. Figure 2 shows a simplified theoretical validation flow chart. 


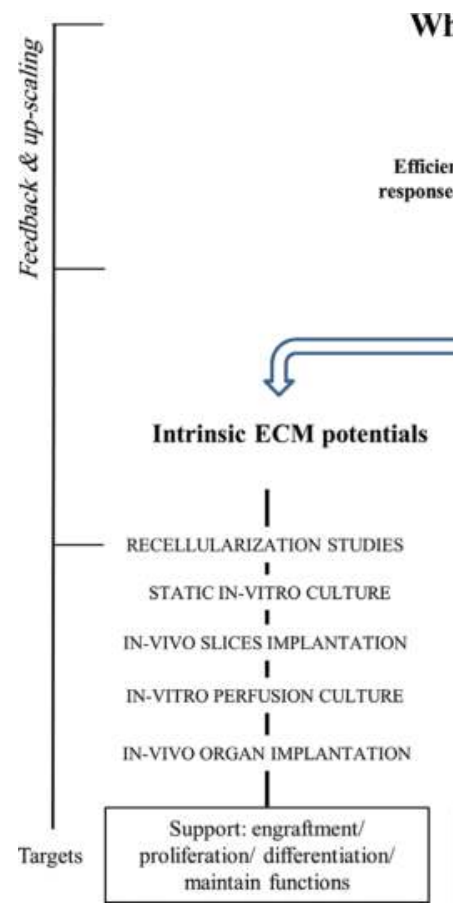

Whole Organ Decellularization

Side-tracks

CELL SOURCES

Characterization

Efficiency/ Bioinductive factors/ Biomechanical/ Host

STERILIZATION

response determinants/Vasculature/ Residual detergents

PRESERVATION

+/-MATERIAL MODIFICATION

$\downarrow$

Testing

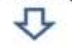

Immunogenicity/ Host

responses

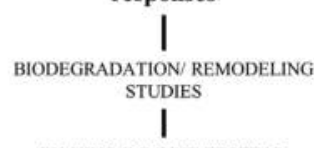

EX-VIVO IMMUNE-TESTING

I

SLICES XENOTRANSPLANTATION

I

ORGAN XENOTRANSPLANTATION

Absent adverse host responses

Dynamic remodeling process

FDA APPROVAL

LARGE-SCALE

PRODUCTION

Figure 2. Schematic presentation of the whole-liver decellularization validation process.

\section{Technique}

To the authors' knowledge, a patent by Anthony Atala in 2002 offered the earliest discussion of the whole-organ decellularization concept [1]. However, Matthiesen et al. [2] presented the first published abstract reporting a perfusion WLD study. Since then, WLD progressed rapidly rendering it a fast growing field. During this progress, a wide range of decellularization techniques have been reported. In this section, the authors focus on the basic principles and significant advances in decellularization techniques in recent years. For a detailed review of the decellularization agents and techniques, the reviews by Gilbert et al. [3], Crapo et al. [4], and He et al. [5] are excellent references.

The decellularization methods can be categorized into chemical, physical, and enzymatic techniques, or a combination of them [3]. Among the chemical agents, detergents are subdivided into non-ionic (e.g., Triton X-100), ionic (e.g., sodium dodecyl sulfate (SDS), sodium deoxycholate (SDC), and Triton X-200), and zwitterionic detergents (e.g., CHAPS) [3]. The most frequently used detergents for WLD are SDS and Triton X-100, which largely replaced the use of peracetic acid (PAA) used widely in decellularized membranes production. Briefly, Triton X-100 causes lipid-lipid and lipid-protein bonds disruption with an adverse effect on glycosaminoglycans (GAGs) content, while SDS acts through the solubilization of cytoplasmic and nuclear membranes, but can result in protein denaturation, and also removes GAGs [3]. 
As a general scheme, the liver is removed after vascular cannulation, and the decellularization protocol proceeds after animal termination. The perfusion of detergents is preceded by saline/ phosphate-buffered saline (PBS)/or deionized water perfusion to wash out the blood and is followed by another step of perfusion (with or without Triton X-100) to wash out the remnant detergents. In between, decellularization agents are perfused utilizing a peristaltic-perfusion pump (with or without pulse dampeners). The perfusion flow rates and durations, the decellularization agents used, their concentrations, and sequences are largely variable [5,6]. Constant flow pressure rather than constant flow rate was infrequently used [7, 8]. Gravity-based perfusion was also reported [9]. An approach of lower SDS concentration with longer perfusion duration or gradually increasing concentration was advocated [10,11], aiming at minimizing the damage to ECM and remnant detergent contents in the decellularized scaffold. In a recent study [12], increasing SDS concentration to $1 \%$ resulted in adverse cell outcomes after cell seeding. Substitution of SDS with ammonium hydroxide is possible [13, 14]. Trends also include the higher reliance on enzymatic techniques as an adjunct to detergents. Gessner et al. [15] incorporated phospholipase and nucleases in their rat decellularization protocol, in combination with $1 \%$ SDC, which yielded efficient decellularization and matrix preservation. Trypsin-EDTA was used in combination in human decellularized liver matrix (hDLM) production [16]. On the other hand, physical methods are only applied in combination with chemical detergents in WLD. Many protocols included freezing/thawing cycles as a step before detergents perfusion to induce cell lysis. Freezing temperatures reach $-80^{\circ} \mathrm{C}$ [13]. Freezing/thawing cycles using liquid nitrogen to room temperature shifts resulted in deterioration of scaffold's collagen content (in an osteogenic ECM) [17].

The decellularization agents are perfused through one of the hepatic vascular systems, most commonly the portal venous (PV) system. To a lesser extent, perfusion through the hepatic veins (HVs) [16], and arterial system (HA) [18], but not the biliary system was investigated. A comparison of portal and arterial perfusion demonstrated lower DNA and better decellularization homogeneity with the arterial route (not statistically significant), while perfusion through the portal vein resulted in higher hepatocyte growth factor (HGF) content [18]. Only one recent study described a technique for in vivo decellularization by surgically isolating one of the rat liver lobes into a separate perfusion circuit [19]. The application of oscillating pressure in a custom-made chamber to mimic the intra-abdominal pressures also resulted in better decellularization homogeneity, lower residual DNA content (not statistically significant), and higher GAGs content (when the arterial system was used for perfusion) [18].

Despite the large number of studies dealing with WLD, the exact quantitative delineation of the effect of any of the decellularization agents remains a difficult task. The use of complex protocols, the characterization at the end of protocol sequence, and the variable detergent concentration, flow rate, flow duration, and characterization panels are some reasons for this difficulty. Designing specific studies for single detergent effects taking into consideration the perfusion parameters is essential; such analysis was employed in kidney decellularization for instance [20]. Thus far, the authors believe that comparative studies are currently the most useful tool in this context. A comparison of Triton X-100 + SDS proved superior to SDS in decellularization of sheep and rat livers in terms of cell removal and ECM preservation, augmented by the results of in vivo implantation [21]. Maghsoudlou et al. [22] demonstrated the impact of pretreatment with EDTA perfusion for $15 \mathrm{~min}$ in a rat 4\%-SDC-based decellularization protocol. The authors 
reported significant decrease of DNA with EDTA pretreatment; the DNA content was, however, $10 \%$ of that in fresh liver. EDTA was more efficient in removing cellular proteins as evidenced by quantitative shotgun proteomics. EDTA pretreatment also yielded a significantly higher content of collagen and elastin, while microarchitecture was more densely packed with reduction in hepatocyte pocket size. This packed microarchitecture could have shared in the higher content of matrix components and rendered the scaffold less suitable for recellularization as concluded by the authors [22]. The use of $1 \%$ SDS resulted in the collapse of vascular network compared to its preservation using 0.5 or $1 \%$ Triton X-100 as demonstrated by corrosion casting [23]. Mattei et al. [24] compared several decellularization protocols for porcine liver slices with or without ionic detergent $(0.1 \% \mathrm{SDS})$; a combination of agitation and immersion was, however, used for decellularization rather than perfusion.

Studies demonstrated the use of livers from various animal species for decellularization including rats [10, 25-27], mice [13], ferrets [28, 29], rabbits [9, 30, 31], sheep [14, 21], and swine $[8,11,32-34]$. Decellularization of whole porcine liver was first reported by Matthiesen et al. [2]. Several reasons render pigs the most suitable organ source for human-scale liver engineering based on the current understanding; reasons include the physiological size matching, rapid maturation, availability for organ harvest, and the possible use of genetic engineering techniques [35]. Wu et al. [36] compared three porcine liver decellularization protocols namely $1 \%$ SDS, $1 \%$ Triton X-100 + 1\% SDS, $1 \%$ SDC $+1 \%$ SDS, all followed by $1 \%$ Triton X-100 in PBS perfusion to remove residual SDS. The authors reported better cellular removal, and higher collagen and GAGs content (70\% of native GAGs) with Triton X-100 + SDS combination. The same protocol also proved to be more biocompatible after perfusion recellularization with primary rat hepatocytes evidenced by significantly higher urea and albumin media content and higher expression of some liver-specific genes. On the other hand, sheep were also advocated for their size matching and anatomy [14]. Kajbafzadeh et al. [14] compared five perfusion protocols for sheep liver decellularization and found that the utilization of ammonium hydroxide + Triton X-100 was the most appropriate in terms of efficiency and intact vasculature. Recently, human liver decellularization in the form of perfusion WLD [16], or immersion/agitation decellularization of liver tissue discs ([37], preprint) was investigated. The source for human livers was either livers rendered unsuitable for transplantation [16] or obtained after hepatic resection for metastatic/benign liver lesions with no underlying chronic disease ([37], preprint). Table 1 summarizes human and porcine liver decellularization studies.

Apart from the decellularization protocol, the wDLM scaffold modification during or after the perfusion process is a continuously growing field. Examples include chemical cross-linking (e.g., formalin) [8], $\mathrm{NaCl}$ matrix stabilization, and heparin immobilization. Cross-linking preserves the matrix structure, interferes with degradation, and masks the antigenic content. $\mathrm{NaCl}$ (as a high-salt buffer) was used to achieve better matrix preservation during the decellularization process [15]. Different techniques for heparin immobilization on the other hand were studied and resulted in decreasing intra-scaffold coagulation in vitro with a relative in vivo vascular patency improvement [7, 15, 32]. Many aspects regarding the effect of these techniques on recellularization, cell-ECM interaction, and scaffold remodeling are, however, still to be elucidated. In a recent study [17], immortalized death-inducible human mesenchymal cells (mesenchymal 


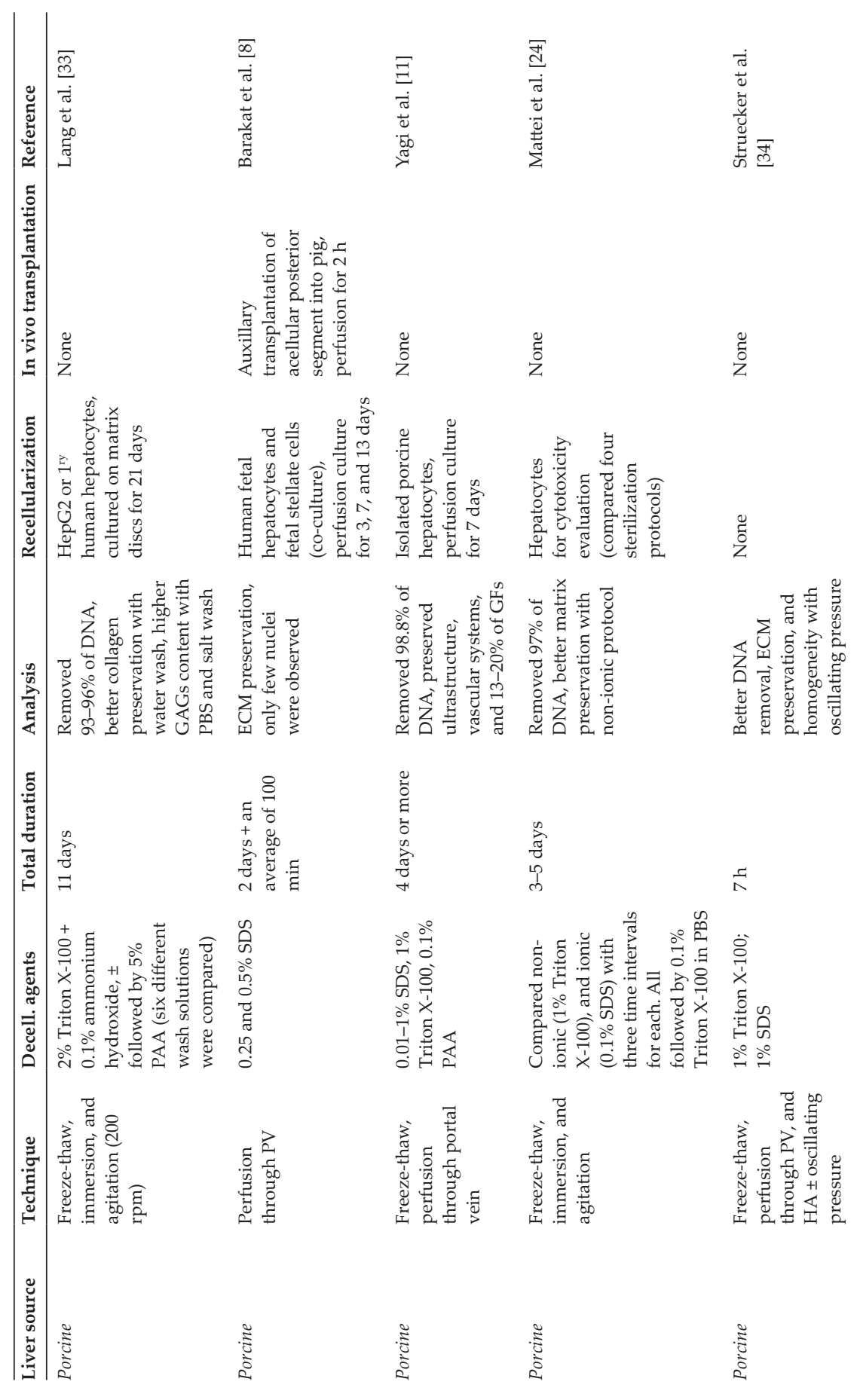




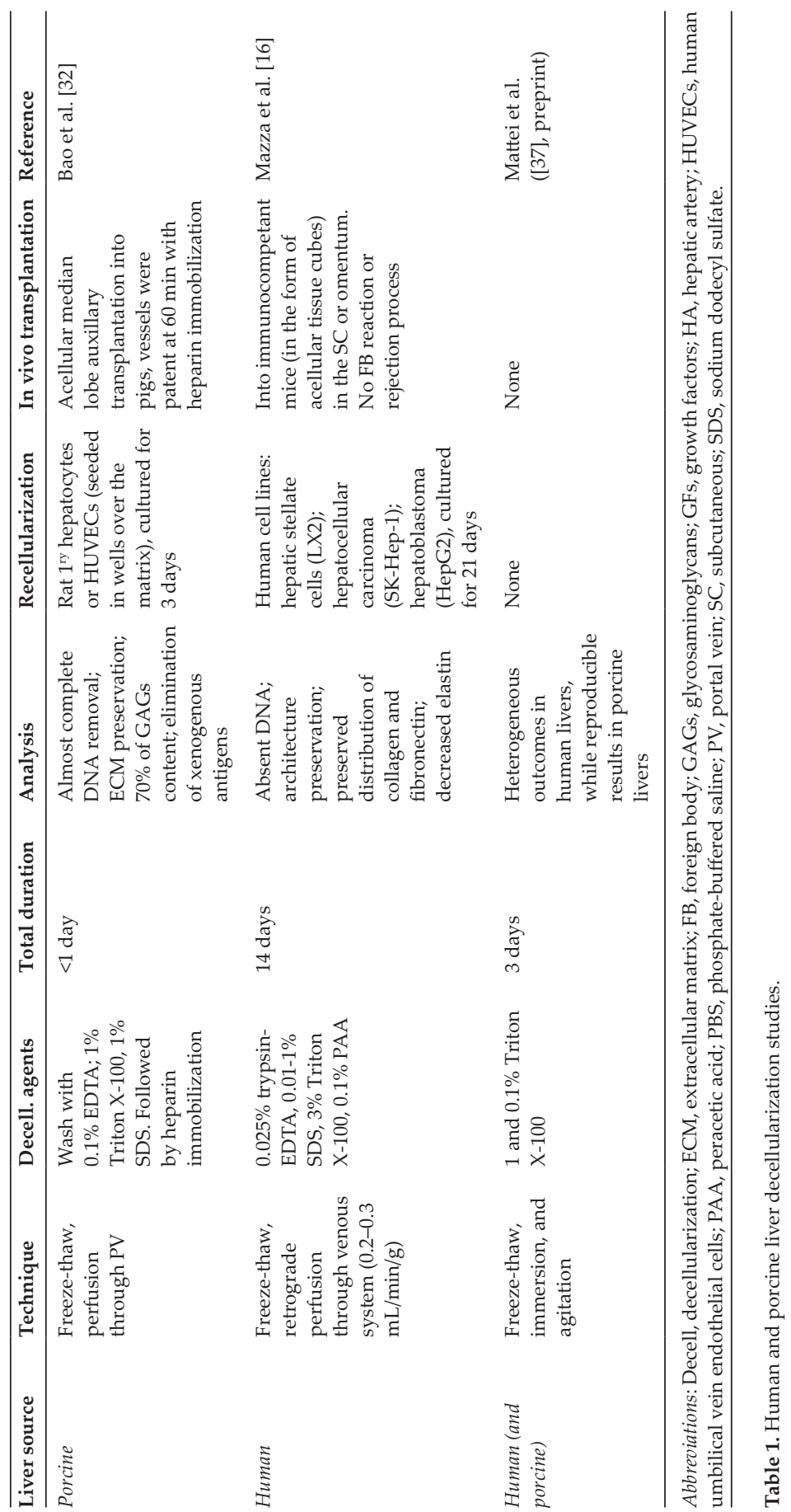


sword of Damocles stromal cell line; MSOD) were used to produce ECM in vitro, and then apoptosis was chemically induced to achieve decellularization. Using MSOD cells overexpressing vascular endothelial growth factor (VEGF) resulted in the production of matrix significantly enriched with VEGF. Applying a similar approach to customize or enforce wDLM composition is not yet investigated.

\section{Characterization}

Defining the outcomes of the decellularization technique is necessary for judging the success of the perfusion protocols and interpretation of the subsequent steps. A standard characterization panel also enables comparison between DLM studies considering the widely variable decellularization protocols. Unfortunately, variation is also evident in the analyzed DLM parameters. For the description of the normal hepatic ECM/biologic scaffolds components and functions, Refs. [38-40] are valuable resources.

The most constant characterization checklist includes matrix and basement membrane structural components (collagen, fibronectin, laminin, and elastin to a lesser extent) and markers of successful decellularization (DNA analysis and absence of nuclear material on histology). Quantitative analysis mostly includes collagen and DNA contents. Accordingly, several protocols resulted in complete preservation of collagen with total/subtotal removal of DNA material; examples include [10, 15, 32]. In case of incomplete removal of DNA, many reports met the criteria previously established for decellularized biological membranes [4]: the absence of visible nuclear material on histological examination using 4',6-diamidino-2-phenylindole (DAPI) or H\&E, the presence of $<50$ ng dsDNA per mg ECM dry weight, and $<200 \mathrm{bp}$ DNA fragment length $[4,41,42]$. Structural components, other than collagens, including elastin [16, 32], reticulin [32], fibronectin, and laminin [24], were less commonly quantified. Few studies showed that elastin was significantly lower in human DLM (hDLM, around 20\%) [16], and rat DLM (20-40\%) [22] compared to controls despite efficient preservation of collagen. Other ECM components that were evidently affected by decellularization protocols are the glycosaminoglycans, with highest preservation around $77 \%$ of native liver [18, 22, 32]. Based on their vital biological roles in cell growth regulations and matrix assembly [43], protocols achieving their maximal preservation are desired. A certain extent of deterioration is, however, reasonable due to their plasma membrane and intracellular components. Regarding growth factors (GFs), an essential component of the bio-functional DLM, Soto-Gutierrez et al. [27] showed the preservation of more than $50 \%$ of hepatocyte growth factor (HGF), and around $40 \%$ of basic fibroblast growth factor (bFGF). Interestingly, Struecker et al. [18] reported a higher level of HGF in rat DLM compared to native liver. Despite this, the exogenous supply of GFs is feasible during the bioreactor-conditioning phase.

The optimal way of comparison between the test and control samples remains a fundamental issue that needs to be addressed and standardized. Normally, cell removal from hepatic tissue results in collapse of the corresponding cellular spaces and consequently a more densely packed ECM on histological and scanning electron microscopy (SEM) analysis. As a result, more intense staining and marker expression can be expected on histological analysis per unit 
area. Mattei et al. ([37], preprint) demonstrated a method for ECM collapse compensation during histological assessment using image analysis. The technique is more easily applicable in porcine liver due to the prominent interlobular septa. Meanwhile, the weight of removed cellular material is compensated by ECM components during quantitative biochemical analysis, resulting in frequently reporting DLM collagen content per unit weight to be higher than native liver, which is obviously an exaggeration. Thus, the accuracy of comparison is compromised for all the quantitative parameters. However, these DLM quantitative analysis figures do represent the true DLM biochemical characteristics as absolute values. For comparison purposes, the use of pre- instead of the post-decellularization weight in quantitative measures can be a valid option, but requires the analysis of the whole liver/liver lobe. Although this can be applicable in small animals, it is not practical for human-sized grafts. Using a weight factor based on the pre- and post-decellularization weights neglects the loss of ECM components and assumes uniformity of decellularization. The authors believe that using predefined anatomical territories or pre-decellularization marked volumes, with pre-decellularization volume-to-weight conversion being a feasible option but lacks validation.

Architectural analysis of the collagen fibers is another crucial aspect of structural integrity beyond mere chemical analysis. It is mostly elucidated using SEM. Satisfactory results were frequently obtained (e.g., $[15,16])$. Validation of complete architectural integrity depends on the appreciation of the delicate ECM organization, which in turn guides cell homing, provides the functional niche for hepatic cell populations, and maintains the hepatic acinus gradation. Mazza et al. [16] demonstrated the preservation of delicate ECM architecture in hDLM including the portal triad micro-anatomy, lobular arrangement, and framework of empty hepatocyte spaces on high magnification. Maghsoudlou et al. [22] measured the size of hepatocyte pockets, a fine refinement for SEM analysis. The authors reported a pocket size of $20.9 \pm 0.5-$ $11.3 \pm 0.3 \mu \mathrm{m}$ depending on the decellularization protocol. The appreciation of the location of ECM components by immunohistochemical techniques relative to normal liver, rather than validating their presence, has become increasingly evident in recent studies and is integral to the concept of hepatic acinus zonation $[15,44]$.

The authors did not observe the utilization of scoring systems as a conjugate to histological techniques and SEM. This can provide quantitative outcomes of decellularization and architectural preservation. On the other hand, scoring systems were developed in case of kidney decellularization. Caralt et al. [45] developed two semi-quantitative scores to compare different kidney decellularization protocols for basophilia and architectural preservation. The scores were applied in relation to glomeruli, tubules, and vasculature. Each component was assessed in 5-10 high-powered fields per histological section. This type of scores does not only reflect the efficiency uniformity throughout the specimen and the differential effect of decellularization protocol on tissue components but also allow for comparison between different studies. The use of image analysis for histological comparison between DLM and native liver ([37], preprint), and the appreciation of decellularization homogeneity [18] were the only close reflections of this concept in WLD.

Surprisingly, Gal epitope, a main barrier to xenotransplantation, is not a frequent characterization parameter in wDLM. In one study, Gal epitope showed remarkable reduction in 
porcine wDLM by immunohistochemistry [32]. The study also demonstrated the absence of swine leukocyte antigen DR-alpha, swine leukocyte antigen-2, Sus scrofa cytochrome B, and porcine beta-actin in DLM compared to native liver. Generally, investigators utilize the DNA content and histological cellular appreciation as markers of efficient decellularization. This does not exclude the presence of antigenic cell components. In fact, Gal epitope was detected in several decellularized matrix products [46], however, with no clear adverse effect on the final remodeling, suggesting a threshold level for adverse outcomes. In view of the short-term graft survival of wDLM in in vivo studies, investigating the contribution of Gal epitope in this cascade is essential. Meanwhile, mass spectroscopy is increasingly being utilized for ECM characterization in recent studies. Recently, White et al. [12] used time-of-flight secondary ion mass spectroscopy (ToF-SIMS) to analyze the surface of decellularized porcine urinary basement membranes; results showed fragment remnants of SDS, Triton X-100, SDC, and cellular material in the form of phosphate and phosphocholine peaks, depending on the decellularization protocol used (with variable dsDNA content). Although these results are protocol dependent and the tissue source is different, the findings propose ToF-SIMS as a valuable tool for the assessment of decellularized materials.

Because the liver is neither a weight-bearing nor a contractile organ, it could be assumed that mechanical properties of the scaffold are not necessary for the physiologic functions of the engineered liver. However, Engler et al. [47] showed that the mechanical properties of the substrate can guide the differentiation lineage of stem cells. Lozoya et al. [48] further investigated the mechano-sensitivity principle on hepatic stem cells; variation in E-cadherin expression in response to alterations in mechanical properties was demonstrated by embedding in hydrogel with different mechanical parameters, suggesting a role of substrate consistency in guiding the cell remodeling and organization. Hepatocytes also showed better viability and function with the use of perfusion bioreactor, or trans-well devices mimicking sinusoidal circulation, when compared to conventional culture. The superior results with dynamic perfusion techniques can, however, be as well explained by better oxygen/nutrients delivery [49-51]. Hsu et al. [52] demonstrated that better hepatocyte viability and functionality are achieved with lower parenchymal pressure in a liver-assist device with a parenchymal chamber design. The biomechanical environment of the liver thus includes the substrate structural properties, as well as the pressures induced by the blood flow and interstitial fluid (although further investigations are necessary to delineate their effects). Another contributor to the hepatic mechanical environment is the intra-abdominal pressures during the respiratory cycle. The increased intra-abdominal pressure during inspiration squeezes the blood through the hepatic veins, and alternatively lower intra-abdominal pressure during expiration results in increased portal venous flow; this alternating cycle of squeeze/aspiration (exemplified as a sponge) was reported to improve the hepatic microcirculation and perfusion $[18,53]$. Therefore, achieving an optimal mechanical environment for cellular differentiation, proliferation, and function relies on both the decellularization protocol, which determines the substrate mechanical properties, and the bioreactor conditioning, which supplies the dynamic component of the mechanical environment. Few studies investigated the effect of decellularization on mechanical properties of DLM. Evans et al. [54] compared the mechanical properties of perfused native liver and DLM of ferrets at the tissue and cellular levels. The study demonstrated a 
significant reduction in the liver stiffness by the decellularization process with a long-term Young's modulus at tissue level of $10.5 \mathrm{kPa}$ in native liver versus $1.18 \mathrm{kPa}$ in DLM, and 4.4 $\mathrm{kPa}$ in native liver versus $0.91 \mathrm{kPa}$ in DLM at the cellular level. Mattei et al. [24] also compared matrix stiffness between native porcine liver and porcine DLM and observed significant reduction in the compressive elastic modulus after decellularization $(1.62 \pm 0.13 \mathrm{kPa}$ for native liver compared to $1.25 \pm 0.07$ and $1.31 \pm 0.09 \mathrm{kPa}$ for two different decellularization protocols). This protocol independency implicates the cell removal as the cause of stiffness reduction, as concluded by the study's authors [24]. Sabetkish et al. [21] demonstrated comparable tensiletesting parameters of rat and sheep wDLM compared to native liver; the maximal load was more similar to native liver when Triton X-100 + SDS were used compared to SDS. The ability to maintain equivalent mechanics in the absence of cells is logically questionable; a more realistic approach can obtain comparable characteristics after recellularization. Characterization related to vascular integrity and infectious potential of ${ }_{\mathrm{WDLM}}$ is discussed later in Sections 8 and 11 , respectively.

In parallel to the standardization of DLM protocols and the characterization of scaffolds' fine constituents, progress in the correlation of DLM parameters with the recellularization, cell behavior, and in vivo outcomes is necessary for efficient feedback tuning. Klaas et al. [55] demonstrated alteration in ECM composition in regenerating liver after liver damage and its contribution in tissue remodeling. The current concept is that maximum preservation of ECM is desired; a deeper understanding based on cell/host-ECM interaction can lead to a tailored approach that can be suitable for the developing liver and fit the cell types used for recellularization. Although tissue sampling is feasible, the development of nondestructive techniques for scaffold characterization is highly desired as the progress continues toward in vivo experimentation. The study by Geerts et al. [56] is perhaps the most relevant in this context, in which the authors used CT and perfusate analysis as nondestructive tools to assess decellularization parameters. DNA levels showed good correlation with the liver Hounsfield unit, and perfusate analysis allowed the assessment of the degree of GAGs' depletion. Nondestructive characterization is also available for vascular analysis. Besides magnetic resonance imaging (MRI) and conventional computed tomography (CT) angiography [21], Gessner et al. [15] reported a nondestructive imaging technique to evaluate the DLM's vascular patency, leakage, and flow rates using ultrasound modalities. The application of this technique to humanscale liver, however, was not yet elucidated. Hagen et al. [57] reported the application of X-ray phase contrast computed tomography (PC-CT) as a nondestructive tool for the assessment of anatomical details of the scaffold and reflected some microarchitectural parameters; the technique is also quantitative.

\section{Intrinsic DLM potentials}

The decellularized matrix potentials can be mainly attributed to its bio-inductive and biomechanical properties [5]. The authors find this functional categorization (bio-inductive/ biomechanical) most suitable for simplification of the whole-organ ECM concept, although the mechanical properties contribute to the bio-inductive potentials as previously discussed. 
The main hypothesis is that the liver ECM-although neither the main nor the viable component of the liver-is able to provide a framework to harness the heterogeneous cell populations of the native liver, maintain their function, and guide their maturation, differentiation, and the graft regeneration process.

Before discussing the evidence and shortcomings regarding this assumption, a major conceptual question should be raised, namely how long should the DLM provide these functions? In vivo studies of ECM-derived membranes for wound healing and tissue reconstruction demonstrated the dynamic interaction between the ECM material and the host environment resulting in initial degradation followed by remodeling. Small intestinal submucosa used to repair canine Achilles tendon was $60 \%$ degraded within 1 month, and complete degradation occurred in a 3-month period as host cells took over ending in successful remodeling [58]. In other words, the decellularized scaffold largely acts as a catalyst and guide for the process of cell migration and initial proliferation, thus a remodeling process can be initiated. In this paradigm, the ECM degradation acts as a release mechanism for bioactive molecules and thus is required for optimal host interaction [38]. However, the concept of biodegradation is not clarified in case of whole-organ scaffolds due to the limitations facing the in vivo experiments. As a result, the fate of the recellularized whole-organ scaffold remains rather vague, taking into consideration that the DLM serves as a transplantable functioning cell reservoir rather than simply guiding the healing process as in case of membrane or tissue repair scaffolds.

DLM-functional expectations include the ability to provide the volume and support for efficient cell engraftment, for a heterogeneous group of cell types (discussed in Section 6), and to grant a bio-inductive environment for their initial proliferation and function (in a bioreactor in case of ex vivo prepping, or in the recipient in case of auxiliary liver transplant). Studies have indeed showed that perfusion DLM proved superior or at least equivalent to regular culture conditions or collagen-sandwich technique in terms of hepatocytic functions and gene expression. For example, immortalized human fetal hepatocytes (IHFHs) showed 2.5-3.5 times increase in mRNA expression of albumin (Alb) and alpha-1-antitrypsin (AAT) after 7 days of culture on DLM compared to regular culture conditions [59].

On the other hand, in vivo studies highlighted the transient functionality of the recellularized DLM (r-DLM). Of significance in this regard are the studies in which seeded DLM slices have been implanted in immuno-compromised animal models, as the adverse host immune response and complex vasculature can be largely excluded, and as a result a more clear appreciation of the in-built DLM potential is achieved. Zhou et al. [59] showed that the activity of IHFH-seeded DLM decreased gradually reaching $2.65 \%$ at 8 weeks using luciferase/bioluminescence in vivo monitoring in immune-deficient mice, which proved superior to both IHFHseeded-Matrigel implants and splenic infusions. In the same study, primary hepatocytes remained viable and functioning on DLM slices in vivo for 6 weeks as shown by green fluorescent protein (GFP) labeling and polymerase chain reaction (PCR) analysis of the explanted scaffolds, where functions comparable to freshly isolated hepatocytes were observed. The change of the primary hepatocytes functions along the 6-week duration was not, however, detailed. Microscopic examination showed the migration of GFP-negative cells into the scaffolds, the nature of which was not also demonstrated. Migrating cells were observed after 
cross-species implantation of hDLM in another study [16]. This time, negative smooth muscle actin (SMA) staining suggested a fibroblastic nature. The temporary function of r-DLM in vivo raises doubts about the inherent abilities of DLM, the core of the WLD hierarchy. A suboptimal construct oxygenation and neovascularization, single-cell-type seeding, and the absent role of positive immune modulation may provide other explanations. The visualization of DLM as a slow release device for bioactive molecules obviously suggests that the inherent stores are gradually drained and therefore required to be replenished by either the seeded cell populations or host contributions in which a positive immune response can be an effector. In this context, the aforementioned fibroblastic infiltration should be considered. It is worth mentioning that different liver cell populations are implicated in the process of ECM production during fibrosis [60]. A study also demonstrated that the behavior of hepatocytes and stellate cells in this regard varied in isolated versus co-culture conditions [61].

The gradient nature of the hepatic acinus ECM is largely overlooked in decellularization experimental studies. Briefly, the ECM components vary from Zone 1 to Zone 3 of the hepatic acinus and therefore provide variable micro-environments for intrahepatic cell populations. Consequently, hepatocytes from different zones showed variation in size and enzymatic functions [62-64]. Zone 1 (periportal) ECM provides the suitable environment for hepatoblastic nature where proliferation is promoted, while Zone 3 ECM (pericentral) promotes cell differentiation. This gradient was evident in ECM components of the space of Disse, in which the Zone 1 ECM resembles fetal/neonatal composition while Zone 3 ECM resembles adult composition [65]. Thus, this delicate ECM gradient allows for corresponding transition of cells from undifferentiated/progenitor cells to hepatoblast-like cells and ending in differentiated hepatocytes toward Zone 3 region. In vitro, ECM components like laminin, collagen III, and collagen IV (Zone 1 components) stimulated cologenic expansion of human hepatic progenitor cells, while cell arrest and hepatocytic differentiation were induced by collagen I which is a component of Zone 3 [63]. The appreciation of zonal gradient should be more evident in the design and interpretation of liver decellularization-recellularization studies. Hypothetically, a whole-liver model should preserve the ability to finely tune the functions of different cell population and therefore has to maintain the differential zonal composition. Gessner et al. [15] showed that Hep3B cell line seeded into decellularized rat livers demonstrated zonalrelated markers expression. Only cells in zone 1 expressed epithelial cell adhesion molecule (EpCAM), which is a marker of undifferentiated/progenitor cells, compared to higher albumin expression in Zone 2 and 3 regions. Characterization of the used rat scaffold also showed the preservation of matrix components in their normal zonal location [44]. Therefore, it can be concluded that delicate decellularization protocols can not only preserve a certain zonal structural gradation but also the respective zonal potentials. The extent of this preservation remains unverified. Klaas et al. [55] also showed that differential changes occur in ECM composition throughout the zonal distribution in response to damage and guide the remodeling process. Establishment of this understanding paves the way for tailored ECM approaches. On the other hand, this gradient of hepatocyte maturation may not only be a function of ECM composition but also has been classically linked to oxygen gradient. In a recent review, Kietzmann proposed interplay between oxygen gradient, beta-catenin signaling, and hedgehog pathway to underlie the classical acinus zonation [66]. 
As aforementioned, studies highlighted the influence of the mechanical properties of the scaffold on cellular behavior and subsequently contribute to the intrinsic bio-inductive arsenal. With this understanding, should an optimum human-scale liver scaffold resemble adult liver regarding its composition and mechanical aspects or rather the fetal and regenerating liver properties? If biodegradation and remodeling is desired, should the DLM preserve almost $100 \%$ of its chemical constituents? Should matrix-stabilizing techniques be used? Answers to these questions remain largely vague. Another issue to be considered is the discrepancy in the ECM composition/architecture between human and animal liver tissue. The porcine liver ECM is most consequential as it is considered the model capable of providing human-scale liver scaffolds. Indeed, the porcine liver is classically demarcated from human liver by its complete interlobular septa [67]; comparative analysis of ECM composition in regard to architecture and zonation is, however, still lacking. Accordingly, differences in interaction of porcine-derived DLM with human-derived cells may exist.

\section{Recellularization}

The ability to repopulate the DLM with human cells is an integral part of the WLD scheme. Animal-derived cells were frequently used to test the WLD hypothesis and improve the techniques. However, the repopulation with human cells is essential for clinical application for immunological and functional reasons. In order to simplify the process, recellularization will be discussed in terms of cell sources, recellularization technique, and optimal cell mass/ density.

The liver is composed of a heterogeneous cellular environment, including hepatocytes, hepatoblasts, endothelial cells (ECs), progenitor cells, fibroblasts, Kupffer cells, undifferentiated cells, and cholangiocytes. An ex vivo-engineered liver should contain all of these cell populations whether by seeding them as differentiated cells or as undifferentiated/progenitor cells along their respective cell lineages and allowing them to differentiate in ex vivo bioreactor setting. In vivo differentiation, although a hypothetical alternative, may lead to blood sequestration issues if the scaffold is insufficiently recellularized due to void intra-matrix spaces.

Different cell sources including cell lines and/or freshly isolated cells were used in recellularization studies to demonstrate the efficiency of the technique, cyto-compatibility of the scaffold, and its intrinsic ability to maintain cell functions or guide the differentiation process. Some of these cell sources are, however, not suitable for clinical application, and others face evident limitations regarding in vitro expansion. As an example, fetal hepatic cells were used to demonstrate the feasibility of DLM recellularization and intrinsic potential of DLM to maintain hepatocyte-specific functions including urea and albumin production $[8,21,29,59,68]$; they are, however, not suitable for clinical application [69] but can be used for liver-support devices [70]. Primary hepatocytes were frequently investigated in recellularization studies [69] and on the functional level are ideal on the background of hepatocyte transplantation research. The main source for primary human hepatocytes is the harvested livers that were found unsuitable for transplantation, which represent a very limited resource [71]. The difficulty with $1^{\text {ry }}$ 
hepatocyte propagation in cell culture is another limitation [72], taking into consideration the minimum number of cells required for clinically valid engineered liver (discussed later). On the other hand, the use of stem cells, whether of embryonic origin (ESCs) or induced pluripotent stem cells (iPSCs), offers several attractive advantages including the capacity to differentiate into various cell lineages and therefore covers the spectrum of parenchymal, vascular and stromal cell components, feasibility of in vitro expansion, and the suitability for patient-specific recellularization approach (in case of iPSCs where the patient's autologous cells can be utilized). However, wDLM recellularization with ESCs and iPSCs, and the potential of DLM to efficiently drive the differentiation into the different cell lineages (with or without differentiation cocktails) remain largely uninvestigated [69]. Despite that mesenchymal stem cells (MSCs) offer a differentiation potential along a more restricted range of cell lineages, they provide a source for stromal components, chemokines, and cytokines. Jiang et al. [13] used bone marrow MSCs to repopulate DLM and showed their hepatic differentiation with hepatocyte-related expression profiles; the recellularized DLMs were able to rescue a model of hepatic failure after their in vivo implantation. The aforementioned cell types can be used alone or in combination to repopulate the liver parenchyma. In case of repopulation with $1^{\text {ry }}$ hepatocytes, the seeding of other cell components is necessary for vascular endothelialization and to reconstitute the hepatic stromal compartment. On the other hand, stem cells may be able to repopulate the three compartments (parenchymal, vascular, and stromal), confirming that these assumptions are, however, still required. Cell sources used for vascular re-endothelialization are discussed in Section 8. Stromal cells including mural cells, stellate cells, and Kupffer cells all share in the hepatic microenvironment, and therefore it is necessary to reestablish them. Baptista et al. [29] showed that the use of fetal hepatic stellate cells with fetal hepatocytes for recellularization is superior to hepatocytes alone. Cells that are not naturally native to human liver may also represent an addition to the recellularization armamentarium. Examples of these cells are the regulatory T-cells (tREGs) that are increasingly investigated for their role in immunomodulation of xenograft rejection and may therefore be a valuable conjugate [73]. A degree of host-cell migration into the wDLM is possible, which can share in replenishing the stromal compartment or the remodeling process. Few studies demonstrated the migration of host cells into the DLM slices, but the nature of these cells was not fully elucidated [32, 74]. Mazza et al. [16] showed the migration of SMA -ve host cells (probably fibroblasts) into human DLM implanted in rats. The fate of migrating host cells and their contribution in DLM remodeling requires further investigation.

Regarding in vivo recellularization, Sabetkish et al. [21] compared in vitro recellularization with in vivo recellularization of rat and sheep perfusion DLM placed in the sub-hepatic region. The authors concluded that in vitro recellularization is superior to the in vivo approach. Histological examination after 8 weeks showed an evidence of angiogenesis, binuclear cells, fibroblasts, and inflammatory cells, with more superior findings with one of the decellularization protocols (Triton X-100 + SDS) and homografts. Liver enzymes were evidently inferior to both in vitro recellularization and native liver [21]. Bao et al. [7] observed a tiny number of PECAM-1 and von Willebrand factor (vWF)-positive cells in non-endothelialized wDLM after in vivo implantation (that lasted $72 \mathrm{~h}$ ). The study's authors suggested that the cells may have extended from the portal system. However, the in vivo approach may not be suitable 
for vascularized grafts unless thrombosis can be efficiently prevented. Vascular thrombosis was reported to occur in $20 \mathrm{~min}$ after non-recellularized DLM transplantation. Blood sequestration in the empty matrix spaces is another concern [32]. In fact, the urgent need for organ replacement in acute liver failure and the debilitated general condition of end-stage liver failure patients make the in vivo recellularization clinical scenario unclear.

The two main recellularization techniques are vascular infusion and direct parenchymal injection. Despite that a report by Shirakigawa et al. [23] showed that directly injected hepatocytes/spheroids (in gelatin) were able to reach a peri-vascular location in the DLM, the vascular pathway was the most commonly used for whole-organ recellularization as it allows for uniform distribution of infused cells and can be suitable for recellularization of all hepatic compartments (parenchymal, stromal, and vascular). Soto-Gutierrez et al. [27] showed better engraftment of cells using vascular perfusion compared to direct injection ( $\geq 67$ and 13\%, respectively). Cell seeding through the portal and hepatic venous systems was the most commonly used with variation in resulting spatial cell distribution. Generally, portal seeding results in higher peri-portal engraftment, while hepatic venous seeding results in higher peri-central engraftment [29]. The simultaneous use of both routes can thus enable better distribution [29]. Hassanein et al. [75] demonstrated that neonatal cell slurry seeded through the biliary tree repopulated the parenchymal regions, CK-7 positivity also marked the presence of cholangiocytes. A comparison between biliary and portal seeding showed that more cells entered the parenchyma with the biliary approach ( $80 \%$ vs. $20 \%$ only) [76]. Regarding the infusion technique, stepwise infusion with periods of 10-15-min static resting intervals has repeatedly achieved better engraftment outcomes ( $\geq 86 \%$ vs. $70 \%$ engraftment, respectively) $[10,11,13,15,27]$, implying the importance of stasis for engraftment. Several perfusion cycles can, however, be required to achieve the desired cell mass.

It is rational that the seeding of whole DLM requires the use of a perfusion bioreactor, as this setting will be mandatory for nutrients and oxygen delivery to seeded cells once the recellularization is undertaken apart from the technique of seeding (bioreactor conditioning is discussed in a separate section). The way the infused cells reach the intra-matrix spaces is yet controversial. Baptista et al. [29] suggested that cell migration occurs through the gaps caused by decellularization detergents that render the vascular wall permeable to infused cells or through selective matrix binding. Interestingly, in a study by Gessner et al. [15], the authors showed that recellularization was successful despite the integrity and non-leakage of the vascular network for $1-5-\mu \mathrm{m}$ micro-bubble contrast material. The authors postulated that cells leave the intact vascular system through a technique similar to hepatocyte transplantation, where migration into the liver parenchyma was shown to occur through sinusoidal endothelial disruption which provides sufficient spaces for cell migration into the parenchyma [77]. Similarly, the authors suggested a sequence of cell attachment and squeeze through the sinusoidal fenestrations [15]. For an overview of decellularized organ recellularization studies, the review by Scarritt et al. [69] is an excellent resource.

Despite the aforementioned advances with recellularization trials, a recellularized liver cell mass/density valid for clinical transplantation is yet difficult to achieve. To understand the recellularization targets, it is necessary to elaborate regarding the minimum requirements for 
clinical transplantation. Thus, the authors believe it is necessary to differentiate between three parameters, namely "functional" cell mass, graft size, and cell density. In a thoughtful review by Caralt et al. [62], the authors suggested that a cell mass equivalent to $5-10 \%$ of host's liver weight is the minimum requirement of scaffold cellular content based on the results of clinical hepatocyte transplantation in patients with acute liver failure. It should, however, be clarified that in hepatocyte transplantation, an intact liver is preserved and transplanted hepatocytes function partially by stimulating liver regeneration besides sharing in the repopulation process (the latter considered as the main mechanism) [78] and therefore may not exactly mimic the seeded-scaffold situation. The aforementioned cell mass was also advocated for acute liver failure and not standardized for all transplantation indications. On the other hand, it is widely accepted as a standard for clinical liver transplantation to use graft weight/recipient weight (GRWR) ratio of $0.8 \%$ as the minimum for transplantation. Meanwhile, the $0.8 \%$ GRWR graft possesses native liver hepatocyte density and functions. Accordingly, a $60-\mathrm{kg}$ individual will require a minimum graft weight of $0.48 \mathrm{~kg}$. Hepato-cellularity assessment showed that human liver contains $139 \pm 25 \times 10^{6}$ hepatocytes/g [79]. Therefore, successful engraftment of 49,920-78,720 billion hepatocytes may be required to achieve comparable hepato-cellularity (compared to approximately 10 billion hepatocytes if a cell mass equivalent to $5-10 \%$ of human liver weight is required), provided that the cell functionality is similar, otherwise a higher number may be necessary. To date, functional parameters of recellularized wDLM equivalent to native liver were not achieved [10, 21]. The authors of this chapter believe that clinical liver transplantation calculations may represent a closer estimation for the whole-organ-engineering requirements and are more generalizable to various transplantation indications in the context of orthotopic liver transplantation. To further augment this, portal implantation of recellularized DLM containing approximately $10 \%$ of total liver cell mass could not achieve long-term survival in 90\% hepatectomized rats $(n=40)$, despite prolonging survival from 16 to $72 \mathrm{~h}$ [7]. The $10 \%$ rule may, however, be applicable if auxiliary liver transplantation or bridging is desired. The short-term failure in [7] can also be attributed to suboptimal function as an alternative explanation.

The second parameter is the optimal cell density. Human liver contains $1.23 \times 10^{8}$ hepatocytes' nuclei per milliliter of liver tissue compared to $1.69 \times 10^{8}$ in rat liver [80]. A study using porcine hepatocytes in bio-artificial liver demonstrated a cell density of $5 \times 10^{6}$ cells $/ \mathrm{mL}$ to be optimal for most of the functional parameters [81]. Interestingly, hepatocytes demonstrated lower functional profiles and viability at low densities, while a density of $5 \times 10^{6}$ or higher was associated with superior parameters in alginate scaffold [82]. Thus far, equivalent densities to native liver could not be achieved considering the inferior cell numbers per gram of recellularized tissue ([7] for example), which can be logically considered to have at least an equal volume to a gram of native liver. The delivery of high concentration of cells can also face technical issues in the form of vascular blockage [62] and insufficient oxygen/nutrients delivery.

Based on the inferior cell density and intra-scaffold function, the graft size in case of recellularized DLM will be consequently larger than that for clinical transplantation. Finally, many parameters regarding recellularization efficiency and substrate potentials require considerable improvement; in addition, the limited cell sources are considered a major hurdle facing the recellularization concept. Dependency on harvested livers deemed unsuitable 
for transplantation as the source for primary hepatocytes is insufficient. Therefore, the development of alternative cell sources including undifferentiated cells/progenitors, the technical refinement of in vitro cell expansion, and bioreactor conditioning is necessary to achieve clinical relevant cell mass.

\section{Immunologic aspects}

The efficient removal of xenoantigens by the decellularization process and thus evading a destructive rejection cascade are imperative to the success of WLD. Repopulation of the scaffold by autologous or allogeneic cells is designed to allow the use of no or routine immunosuppressive therapy, respectively. The oligosaccharide $\alpha$-Gal (Gal $\alpha 1,3-G a l \beta 1-4$ GlcNAc-R; Gal epitope), which is mainly found as a cell membrane antigen, and xenogeneic DNA are considered the main antigens stimulating the rejection cascade for xenogeneic biomaterial [46].

Studies showed that commercially available decellularized biologic scaffolds and prosthesis retain a variable amount of Gal epitope. An increase in anti-Gal antibody levels was also noted after transplantation of xenogeneic material [83, 84]. The use of galactosidase resulted in a decrease in T-lymphocytic infiltration in porcine cartilage grafts [84]. Ex vivo exposure of small intestinal submucosa (SIS) of porcine origin to human plasma showed predominant IgG2 fraction conjugation; Gal epitope was shown to stimulate the same fraction [85, 86]. However, complement activation did not occur, probably due to the low density of the epitope [87, 88], suggesting a threshold for adverse outcomes. Raeder et al. [89] showed that the implantation of SIS in Gal knockout mice resulted in the formation of anti-Gal antibodies; furthermore, pre-sensitization with sheep erythrocytes resulted in more intense early inflammatory cellular infiltration. Despite that these findings highlight the retention of a variable amount of Gal epitope after tissue processing, the host response did not affect the final remodeling outcome. On the other hand, analysis of commercially available ECM products also showed the presence of DNA material $[90,91]$. Although remnant DNA was shown to drive an inflammatory process, the clinical success of these materials implies that adverse host responses are also unlikely to occur below a certain threshold amount of DNA fragments retained. The ECM biodegradation process should normally include the remnant DNA content as well [46]. Crapo et al. [4] suggested criteria for remnant DNA that are necessary to avoid an adverse inflammatory/immune response and therefore allow graft remodeling; these criteria are the absence of visible nuclear material on histological examination using 4',6-diamidino-2-phenylindole (DAPI) or H\&E, the presence of $<50$ ng dsDNA per mg ECM dry weight, and $<200$ bp DNA fragment length [4,41,42]. These criteria were frequently met in WLD studies. A recent study demonstrated complete removal of DNA from human DLM [16]. However, Gal epitope analysis is not routinely included in DLM characterization panel as aforementioned. Bao et al. [32] demonstrated remarkable reduction in Gal epitope compared to native liver by immunohistochemical staining. Although both the consistent efficiency of the decellularization outcome ([37], preprint) and the complete removal of all cellular components [4] are debated, the current decellularization parameters of wDLM were enough for the clinical success of the decellularized biologic membrane scaffolds [4]. 
Transplantation of porcine organs into primates results in rapid innate immune response driven by natural antibodies, which bind to vascular endothelium and result in complement activation and hyper-acute rejection (HAR). An innate cellular response comprising macrophages, neutrophils, monocytes, and natural killer cells coexists. Innate cellular immune response results in the development of a delayed form of rejection commonly named acute vascular rejection (AVR) if HAR was successfully evaded [35, 73]. The extent of adaptive immune response and the contribution of T-cell in acute cellular rejection (ACR), a classical component of allo-response, are, however, controversial [73].

Few in vivo studies have examined the immunologic response to perfusion DLM slices across species. The first [74] examined a pig DLM to rat xenotransplantation model and rat DLM to rat allotransplantation model, where DLMs were implanted in the subcutaneous dorsal adipose tissue. The specimens showed no capsulation, exudation, or a noticeable adverse host response in the adjacent tissue in both models along 28 days. The total WBCs count did not show a significant increase as well over 28 days. Although cellular infiltration was evident after 7 and 28 days, low to no $\mathrm{CD}^{+} \mathrm{T}$-lymphocyte activation was noted, the infiltrating cells showed positivity for the pan-macrophage marker (CD68), but neither M1 nor M2 phenotypic markers were evident. The second [16] examined a human DLM to rat xenotransplantation model. Cubic DLM fragments were implanted both subcutaneously and in the omentum. Mild inflammatory response was observed in the surrounding tissue after 7 days in the form of polymorphonuclear (PMN) cells and lymphocytes infiltration, while reduced or no inflammation was detected after 21 days. Analysis demonstrated predominance of CD3+ T-cells. SMA -ve cells (probably fibroblasts) were observed at the time of explantation. In both aforementioned studies, scaffolds were well characterized regarding the DNA content and structural components, and no adverse immune response was observed. On the other hand, Sabetkish et al. [21] reported inferior results of in vivo recellularization of xenografts (sheep to rat) compared to homografts (rat to rat) after sub-hepatic implantation of DLMs; xenografts also showed more marked inflammation and fibrosis.

It is important, however, to highlight that the biological membrane and in vivo DLM slices studies may not accurately mimic the immune response to bioengineered whole-organ transplants for four reasons: (1) the techniques of decellularization and processing of biological membranes differ from that for DLM; therefore, host responses can vary. A comparison of host responses to five different ECM products-four of them were xenogeneic-showed a considerable variation in host response, explained by their different processing techniques $[46,92]$. (2) Studies demonstrated the difference in immune response between vascularized and non-vascularized grafts represented in the antigen immune dominance and the strength of indirect allo-response $[93,94]$. Although these findings are related to HLA antigens, which have less/no significance (apart from the current debate) in clinical liver transplantation and obviously no direct link with decellularized material, a discrepancy in the response may still exist. The presence of Gal epitope on vascular endothelial cells is in fact considered the main drive for the hyper-acute rejection of xenotransplants [46, 95-97], and this cannot be elucidated using DLM slices. The three previous studies used non-vascularized grafts and thus the classical sequence of HAR and AVR (the main pivots of xenotransplantation rejection 
cascade) could not be examined. (3) Transient immune/host response considered as benign in the aforementioned studies can be sufficient to induce vascular/sinusoidal thrombosis/blockage in the vascularized model. In vivo studies of xeno-response to vascularized DLMs are still missing. The last two assumptions are yet impossible to investigate due to the short-term graft survival in wDLM transplantation trials, taking into consideration however that an immune/ inflammatory response can contribute to this short-term failure. (4) Finally, despite the fact that human-to-rat, pig-to-rat, and sheep-to-rat models are xenotransplantation models by definition, their relevance to pig-to-human xenotransplantation model is largely questionable. The aforementioned xenotransplantation models do not represent Gal-positive to presensitized Gal-negative transplantation models [35]. It would be very interesting to elucidate the immune response in a complement-enriched, pre-sensitized Gal-knockout or non-human primate (old world monkeys) recipient after vascular anastomosis.

Two important approaches should be mentioned in this context because of their capability to largely bypass the host/immune response to xeno-grafts dilemma if all the other parameters were optimized. The first is using human livers for the decellularization process; livers that are found unsuitable for transplantation are good candidates for this approach [16]. However, despite the structural and immunological advantage, this approach does not massively expand the organ pool. The second is using the native liver for an in vivo decellularization process. In a very interesting study, Pan et al. [19] showed the possibility of in situ liver decellularization by constructing a separate perfusion circuit in vivo for one of the rat liver lobes and using it for the decellularization and recellularization sequence. This may represent the optimal approach regarding the immunological aspect and organ conservation; however, it requires healthy ECM and structural integrity (and thus excludes malignancy, cirrhosis, and biliary atresia for example as an indication for transplantation). Also, many parameters should be addressed in the ex vivo setting before transferring them into an in vivo model. Both models will not be further discussed in order to keep the context of xenotransplantation.

\section{Vasculature}

An intact vascular network able to convey oxygen and nutrients to the deeply seated heterogeneous cell populations is perhaps the most attractive feature of wDLM scaffolds due to the difficulty of the artificial imitation of such complexity. Intact vasculature is essential for the transition of ECM applications from membranes/slices to the complex 3D organ format. Meanwhile, thrombus formation and blockage of the vascular network is largely adopted as the explanation for the repetitive failure of $\mathrm{wDLM}$ in vivo experiments, even when heparinization is employed.

The liver contains three vascular networks namely the portal venous system, the arterial system, and the hepatic venous drainage, in addition to the biliary system. Although all the routes except for the biliary system have been utilized for perfusion decellularization, the portal venous system remains the most widely used cue for both decellularization and recellularization as discussed previously. Ideal characteristics of the vascular system after 
decellularization include the following overlapping parameters: full integrity, sustainable patency, non-thrombogenicity, ability to withstand blood pressures within the physiological ranges without leakage or rupture, complete decellularization, and efficient recellularization. Most decellularization studies include tests for vascular integrity as a part of their characterization panel, including corrosion casting [8, 10, 25, 27], CT angiography [21], fluoroscopy [9, 28, 29], dye injection, MRI [21], ultrasonography modalities, and confocal microscopy [29], or a combination of them $[8,29]$. SEM on the other hand is frequently used to demonstrate the vascular architecture and the inter-vascular relations. However, characterization is sometimes restricted to the portal and hepatic venous system as it is the route used for perfusion and presumptively suffers the maximum burden of the decellularization reagents. Nondestructive approaches (e.g., angiography, fluoroscopy, MRI, and ultrasonography) that are applicable in human-sized liver model are more suitable for the clinical approach. The ability to demonstrate leakage, besides structural integrity, is also important [15]. An intact DLM portal venous system has been sufficiently demonstrated in several reports. Gessner et al. [15] demonstrated the patency and integrity of DLM portal/hepatic vasculature using 1-5- $\mu$ m micro-bubble contrast agent, which did not leak into the scaffold matrix. An advantage of this technique is the validation of sinusoidal compartment integrity. The patency of the arterial and biliary systems has been less frequently demonstrated using the corrosion-casting technique [10, 27]. Their patency/integrity is, however, necessary for clinical application. Although the combination of corrosion casting with SEM can offer some quantitative parameters for the vascular tree [98], the ability of corrosion casts to demonstrate leaks through minute gaps in the vascular wall is questionable.

Blockages by cellular elements or thrombosis can interfere with vascular patency in vivo. The prevention of in vivo occlusion/thrombosis is, however, a complex task and is currently viewed as the bottleneck for the progression of wDLM in vivo experimentation. Herein, the authors try to dissect the confounding factors of this adverse outcome. For the decellularized vessels to be non-thrombogenic, two conditions need to coexist: (1) efficient decellularization/antigen removal, with successful evasion of intravascular inflammation, HAR, and AVR cascades. An inflammatory response with leukocyte recruitment can act as the nidus initiating thrombus formation. Acute rather than chronic inflammation was associated with an increased risk of venous thrombosis [99]; and (2) efficient vascular re-endothelialization. Thus, the exposure of underlying matrix components that can provoke platelet adhesion/aggregation ending in thrombus formation can be prevented. It is thus important herein to refer to the classical Virchow triad of venous thrombosis, including stasis, changes in vessel wall, and blood changes. Incomplete recellularization results in considerable empty matrix spaces and therefore permits pooling of blood and stasis. On the other hand, a suboptimal vascular endothelium falls under the "vessel wall changes" component along with the actual vessel wall damage by the decellularization protocols. Appreciation of the functional parameters of seeded endothelium along with the seeding efficiency, viability, cell attachment, and endothelial distribution is essential. Of special significance is the expression of anticoagulant proteins, which can again be affected by hypoxia and inflammation [99]. Robertson et al. [100] described the application of an ex vivo thrombomodulin assay to assess the anticoagulant functions of seeded endothelium in decellularized heart scaffold. This is achieved through perfusion of 
human alpha-thrombin and protein $C$ followed by the assessment of thrombomodulin and thrombin-mediated protein C activity. Seeded scaffolds showed six- to eightfolds significantly higher thrombomodulin and thrombin-mediated protein $\mathrm{C}$ activity compared to acellular ones, signifying their capability of coagulation cascade inhibition. Further investigations of the seeded-endothelial-functional profile in wDLM are necessary.

In case of small-caliber decellularized vascular grafts, an animal study demonstrated the patency of most of the small-caliber (1.5-mm inner diameter) decellularized arterial xenografts (without pre-implantation endothelialization) after 4 weeks when used for the repair of carotid arteries [101]. The patency of 4-mm-diameter decellularized vascular grafts was improved by surface heparin treatment resulting in only $8 \%$ thrombosis after 6 months in another study; vessels also showed efficient in vivo cellular migration and remodeling [102]. On the contrary, in vivo implantation of non-recellularized porcine DLM resulted in complete vascular occlusion after only $20 \mathrm{~min}$, compared to preserved patency at $60 \mathrm{~min}$ when a heparin immobilization technique was used [32]. Several factors including the smaller vascular diameters, the length of the vascular tree, and blood flow dynamics inside the scaffold (e.g., flow rate and turbulence) can result in this discrepancy when the wDLM and decellularized vascular xenograft models are compared. Complete vascular recellularization at the time of implantation is therefore necessary in wDLM [69]. Baptista et al. [29] performed ex vivo blood perfusion in ferret $\mathrm{wDLM}$ with or without endothelial cell seeding and reported significantly less platelet adhesion in seeded scaffolds. Human umbilical vein endothelial cells (HUVECs)seeded scaffolds also demonstrated less leakage compared to non-seeded scaffolds in another study [23].

For parenchymal recellularization, choosing the appropriate cell sources, seeding technique, and maturation process design are paramount to optimal vascular re-endothelialization. Basically, autologous cells should be used for vascular recellularization if a non-immunogenic DLM is the target. However, using allogeneic cells is another option with the use of immunesuppressants, with reference to clinical transplantation. The cells used should possess a considerable proliferative capacity in vitro to allow the repopulation of the entire hepatic vascular surface area. Therefore, other alternative cell sources besides endothelial cells are needed. Bone-marrow MScs, iPScs, and progenitor cells were suggested for endothelialization of decellularized vascular grafts [103]. Another desired characteristic is the ability to differentiate into the different vascular wall components (mainly ECs and smooth muscle cells "SMCs"); otherwise, they should be supplied independently. Interaction with endothelial ECM was demonstrated to guide the endothelial differentiation of MSCs without other stimulants [104]. MSCs from bone marrow and adipose tissue also possess the ability to differentiate into SMCs, rendering MSCs an attractive option for vascular recellularization [105, 106]. The review by Bajpai et al. [103] is an excellent review of stem cell sources in vascular graft engineering.

ECs of different sources were the only cell type investigated for wDLM re-endothelialization. Uygun et al. [10] used microvascular ECs to seed rat DLM previously recellularized by hepatocytes; the ECs were seen lining the vascular elements after 3 days of culture. Baptista et al. [29] seeded ECs into ferret DLM; although evidence of vascular coverage was noted, technical 
limitations did not allow the confirmation of complete coverage of the vascular system. In the same study, seeded HUVECs through portal vein showed a distribution of capillary pattern around larger vascular structures. Shirikagawa et al. [23] also demonstrated the seeding of HUVECs into rat DLM. The seeded HUVECs despite not leaking outside the vascular system and attachment to internal surface of vascular element, they were only observed in a limited cavity. ESCs and iPSCs were used for vascular recellularization of other decellularized organs but not DLM [69]. Infusion recellularization is the technique generally adopted for DLM endothelialization. Interestingly, Ko et al. [107] reported a two-step endothelialization technique comprising a static and dynamic phases starting with the static component. The technique resulted in efficient re-endothelialization with the use of antibody conjugation to DLM. The portal vein is the route commonly utilized for this purpose [10, 23, 29]. As previously mentioned, Baptista et al. [29] compared the portal and hepatic venous recellularization approaches for epithelial cells and concluded that using multiple routes can result in better scaffold recellularization. Ko et al. [107] simultaneously used inferior vena cava (IVC), PV, and HA for vascular re-endothelialization of porcine DLM but without comparison to perfusion through a single route.

After the process of vascular recellularization, a period of in vitro maturation in a dynamic bioreactor design is necessary for cell differentiation, expansion, production of ECM, and remodeling before implantation. For decellularized vascular grafts, the maturation can be achieved in 3 weeks [108], in which exposure to physiological cyclic pressures inside the bioreactor setting is a constant core concept.

Bao et al. [32] applied heparin immobilization to a well-characterized porcine whole DLM using three different techniques. End-point attachment technique proved to be the most efficient. Interestingly, thrombosis did not occur in heparin-immobilized DLM after the auxiliary transplantation of the median lobe into pigs, and blood flow continued for 60 min though not endothelialized compared to $20 \mathrm{~min}$ in control DLM. The vessels proved to be patent by histological examination at explantation. However, it was not clear why perfusion was not continued after the 60-min period. Also, the arterial system was not anastomosed and the in vitro study showed that heparin is released from the scaffold to reach $3.6 \%$ at the seventh day. The authors concluded that heparin immobilization can boost the anti-thrombogenic nature of DLM and showed that it did not interfere with cell seeding. The effect of heparin immobilization on endothelial cell seeding is, however, unknown. The study also highlighted the discrepancy between the outflow and inflow rates, which the authors explained by sequestration of blood inside the DLM. This finding highlights the need for efficient parenchymal recellularization before transplantation, as stagnation of blood in the suboptimally recellularized matrix zones can also promote intra-parenchymal blood clotting and interfere with oxygen and nutrient delivery.

In a trial to maximize endothelialization of wDLM, Ko et al. [107] used anti-endothelial antibody conjugation to porcine wDLM, coupled with endothelial cell seeding. The authors reported efficient re-endothelialization of $80-90 \%$ of the extra-capillary vasculature evidenced by green fluorescent protein utilization. The endothelialized scaffolds showed three- to fourfolds lower platelet adhesion in vitro and maintained superior vascular patency $24 \mathrm{~h}$ after in 
vivo transplantation. Lastly, the mechanical strain effected by the blood flow and pressure within the physiological ranges represents part of the mechanical environment of the liver [62]. As aforementioned, mechanical factors can influence the cell behavior and affect the hepatocytic and endothelial cell functions. Therefore, imitation of these physiological pressures in vitro in view of the dual nature of hepatic blood supply can have an impact on vessel wall acclimatization and cell functions.

\section{Bioreactor conditioning}

Once a whole-liver scaffold is recellularized, the use of a perfusion culture is necessary to allow nutrients and oxygen delivery to the depth of the scaffold. The term bioreactor is commonly used to describe the perfusion culture setting that should also include an oxygenation system, bubble trapping, and allow for media replacement and sampling. The bioreactor does not only serve as a temporary viability maintainer till transplantation but is incorporated in many study designs as a station for scaffold preparation and optimization. The bioreactor setting also provides a 3D in vitro culture model to assess the different parameters of cell-ECM interactions.

An optimal scaffold/bioreactor combination should be capable of supplying the most suitable microenvironment for cell proliferation and differentiation of stem/progenitor cells along the hepatic lineages. Defining the parameters of the optimal environment and developing the appropriate techniques to achieve them are thus necessary; neither, however, is a simple endeavor. Mimicking the native human liver perfusion dynamics is generally visualized as most appropriate for mechanical acclimatization and optimal cell functionality. Briefly, the liver receives $75 \%$ of its blood supply through the PV with a low flow pressure $(4-10 \mathrm{mmHg}$, nonpulsatile flow), low $\mathrm{pO}_{2}(30-40 \mathrm{mmHg})$, and carries nutrients absorbed from the intestines. The HA supplies the other $25 \%$ of blood supply with a flow pressure around $120 \mathrm{mmHg}$, high $\mathrm{pO}_{2}$ (90-100 $\mathrm{mmHg}$ ), and a pulsatile flow pattern [62]. Apart from the technical difficulty to achieve such a complex dual perfusion pattern (considering the single outflow and the differential $\mathrm{pO}_{2}$ content at least), the suitability of these parameters for the developing recellularized graft is questionable for several reasons: (1) in the absence of complete endothelial barrier, the shear stress resulting from the high flow pressures can have adverse effects on the hepatocyte viability and function [62]. (2) These defined flow parameters may not be the optimum for the developing liver tissue, in which higher proliferation and maturation activity are expected, or in case of recellularization with stem/progenitor cells. For example, the developing fetal liver receives its blood supply from the umbilical vein, the PV (low oxygen and nutrient content), and the HA, with differential contributions to the right and left hepatic lobes, where portal vein supplies only the right lobe [109]. Therefore, a design based on the cell/target-specific requirements can be more appropriate at the developing stage than mimicking the developed liver parameters. (3) Mimicking natural flow parameters in the perfusion system does not guarantee mimicking natural equivalent values at the cell level due to the discrepancy between the intra-hepatic flow pattern in the decellularized/recellularized graft (e.g., turbulence) and the native liver, besides the use of artificial oxygen delivery modalities. Nishii et al. [110] studied the mechanical 
micro-environment in decellularized versus native ferrets liver in an ex vivo setting with portal perfusion (flow rates $3-12 \mathrm{~mL} / \mathrm{min}$ ), and integrated the data into multi-scale computational model. Decellularization resulted in $82 \%$ decrease in vascular resistance with mean fluid pressures of $0.6-2.4 \mathrm{mmHg}$ and mean velocities of $250-840 \mu \mathrm{m} / \mathrm{s}$ along four different studied flow rates. The authors also reported a 5.6 times increase in hydraulic conductivity as a measure of tissue permeability in decellularized livers. These findings are of great relevance to the process of perfusion recellularization and bioreactor conditioning. However, further similar studies for recellularized livers are desired as the seeding process can expectedly impact the vascular flow dynamics. In fact, Bao et al. [7] reported portal hypertension and ascites after portal implantation of recellularized grafts rather than decreased vascular resistance, which can result from the seeding process (hepatocyte spheroids in this case). Also, the arterial flow dynamics and the application of similar computational methods in porcine liver need to be explored.

In order to evade high shear stress, most studies adopted a sub-physiological PV flow rate ranging from 0.5 [29] to $15 \mathrm{~mL} / \mathrm{min}$ [10]. The duration required for conditioning is a function of the cell source/mass and the desired degree of recellularization. More than 1 month can be needed for stem cell differentiation [62]. Decellularized vascular grafts on the other hand require a period of 2-3 weeks for maturation [108]. Despite the development of hepatocytespecific functions and hepatocyte-related gene expression in the perfusion culture setting comparable to levels in collagen-sandwich culture, they only represented 20 and 30\%, respectively, compared to in vivo levels of albumin production and gene expression [10]. Such subphysiological levels can be explained by either an inferior cell-cell and cell-ECM interaction or an unsuitable bioreactor setting. The failure of a heterotopic rat-transplant model containing around $10 \%$ of liver total cell mass to support long-term survival despite sustained native portal perfusion after $90 \%$ hepatectomy fortifies the first possibility.

Studies of hepatocyte hypoxia and oxygen tension at cellular level rather than cell viability are needed. Oxygen delivery methodology should also be reevaluated. The development of nondestructive modalities for monitoring cell parameters inside a bioreactor is necessary for continuous troubleshooting. Ren et al. [111] developed a nondestructive technique to assess cell viability in decellularized whole organs based on a resazurin reduction perfusion assay. The application in decellularized rat lung seeded with endothelial cells showed good matching with histology and interestingly showed no significant difference between constant flow rate $(2 \mathrm{~mL} / \mathrm{min}$ ) and gradually increasing flow rate (from 2 to $8 \mathrm{~mL} / \mathrm{min}$ ) along 7 days.

\section{Vascularized DLM in vivo studies}

The transplantation of recellularized DLM (vascular/parenchymal) across a physiologically relevant xenotransplantation model through vascular anastomosis is the best tool to collectively test the whole-liver decellularization hypothesis. However, reasons for adverse outcomes in this setting consequently can be multifactorial. A limited number of studies investigated the in vivo vascularized graft survival/function or vascular patency using different combination of the aforementioned parameters. Uygun et al. [10] reported a rat-to-rat heterotopic transplantation 
of recellularized DLM (1 $1^{\text {ry }}$ hepatocytes only) using arterio-venous anastomosis for portal flow reconstruction, with harvest after $8 \mathrm{~h}$. Despite retaining the location, morphology, and some functional markers of hepatocytes, the authors observed minimal damage to hepatocytes due to the shear stress caused by arterial flow and minimal ischemic damage. No comments were offered regarding the vascular tree patency and platelet adhesion; anticoagulation was, however, used. Bao et al. [32] reported heterotopic pig-to-pig transplantation of decellularized median lobe (without recellularization) to assess the effect of scaffold heparin immobilization in preventing vascular thrombosis; the portal inflow was established using the left renal vein. Complete vascular thrombosis occurred in controls after $20 \mathrm{~min}$, while patency was sustained for $60 \mathrm{~min}$ in case of heparin immobilization. The immunologically relevant status of the donor/recipient match was not elucidated in the aforementioned studies. Bao et al. [7] described a rat-to-rat (inbred) heterotopic recellularized DLM transplantation (hepatocyte only); the DLM was modified by layer-by-layer heparin immobilization before cell seeding. The recipients were $90 \%$ hepatectomized before transplantation of the graft, which was implanted in continuity with the portal vein. After $72 \mathrm{~h}$, the hepatocytes maintained morphology, organized into aggregates with vessellike structures formation, and expressed several liver-related genes and liver-specific functions with minimal apoptosis and detected proliferation marker (BrdU). The authors observed a tiny number of PECAM-1- and vWF-positive cells that may have extended from the portal system. Rats also developed ascites and gastrointestinal congestion due to portal hypertension, which was explained by the authors as small-for-size syndrome. Although the heterotopic liver slowed down the rise of ammonia level and was able to significantly prolong the survival time after hepatectomy from 16 to $72 \mathrm{~h}$, it did not allow for long-term rat survival. The authors did not comment on the extent of vascular thrombosis in this transplantation scenario. However, in the same study, non-recellularized DLM with heparin immobilization was thrombo-resistant for $3 \mathrm{~h}$ after heterotopic transplantation. Two very important inferences of this study are (1) if the vascularized DLM can be able to function long enough without thrombosis, it is probable that endothelial cell ingrowth from the nearby vasculature can occur as suggested by the authors [7], a similar fashion to which occurs in decellularized vascular grafts may apply, and (2) the hurdles against in vivo application are not restricted to vascular patency; the function capabilities of recellularized vascular grafts in vivo were also non-optimal (taking into consideration that the graft contained a mean of $10.65 \%$ of the whole rat liver cell mass as evidenced by DNA content). Surprisingly, except for the last study, the rationale behind the time frame of the in vivo study was not clearly elucidated. Bruinsma et al. [112] also examined the layer-by-layer heparinization technique. The authors confirmed that recellularization with primary rat hepatocytes was not affected by the process. The heparinized grafts showed no visible clots and better flow during ex vivo perfusion. However, after heterotopic transplantation, the heparin layering did not improve the flow or transplantation outcomes despite lower evidence of thrombosis. A main difference between the last two in vivo studies with heparin immobilization is the $90 \%$ hepatectomy performed in the former one, which may have affected the coagulation profile.

Ko et al. [107] on the other hand reported in vivo implantation of efficiently endothelialized porcine DLM making use of antibody conjugation technique. The grafts demonstrated evidently better patency and lower platelet adhesion compared to acellular grafts. Table 2 summarizes the trials of vascularized DLM transplantation. No immunological studies of vascularized DLM 
transplantation yet exist. Further in vivo transplantation studies are highly desired for both isolated and combined parameters. The advances in recellularization and the upgraded understanding of the underlying scaffold and blood-flow mechanical contributions pave the way for in-depth analysis of in vivo experiments.

\section{Sterilization}

A prerequisite for the success of WLD is the efficient elimination of infectious potential of the scaffold to prevent xenosis. The absence of cellular content allows the use of sterilization techniques, since no concerns regarding cellular damage exist. However, validation of the efficiency and cytotoxic effects of individual sterilization techniques is necessary for recellularization and in vivo progress.

Techniques used for sterilization of acellular scaffolds include ethylene oxide (EO), peracetic acid, and ultraviolet and gamma radiations (GRs). Kajbafzadeh et al. [14] compared six different sterilization protocols on sheep DLM including EO, GR, freeze-drying (FD), EO + GR, FD + GR, and PAA + GR. Interestingly, the protocols utilizing a single agent did not achieve efficient sterilization compared to full sterilization by combination protocols. However, combination with FD resulted in inferior mechanical outcome, while EO + GR and PAA + GR had no mechanical influence. Mattei et al. [24] investigated the cytotoxic effects of three sterilization protocols on porcine DLM, including PAA, exposure to chloroform gas, $\mathrm{H}_{2} \mathrm{O}_{2}$ gas plasma, or a combination of the last two agents, all after FD. The study identified PAA and chloroform gas as the best agents in terms of cyto-compatibility.

Bao et al. [32] investigated the effect of decellularization protocol on the infectious potential of porcine DLM. Interestingly, the DLMs were devoid of porcine endogenous retroviruses (PERVs), a major concern in porcine grafts, and PERV polymerase compared to native liver. Sarikaya et al. [113] demonstrated the antibacterial activity of ECM extracts derived from porcine small intestinal submucosa and urinary bladder submucosa against Gram-positive Staphylococcus aureus and Gram-negative Escherichia coli. Extracts were able to inhibit bacterial growth for at least $13 \mathrm{~h}$. Antibacterial and antifungal medications can also be added to the matrix during the process of perfusion to improve sterilization.

\section{Large-scale production}

It is rational that the success of the clinical trials should be achieved before seeking largescale production of on-shelf wDLM scaffolds. However, in this section, the authors try to delineate the parameters highlighted by previous studies along the pathway to on-shelf commercialization.

Animal source: a consensus regarding the most suitable animal species and age for organ harvesting was not yet reached. However, the most prevalent opinion favors the use of porcine liver. Despite the previously outlined discrepancy in ECM structure between human and porcine 


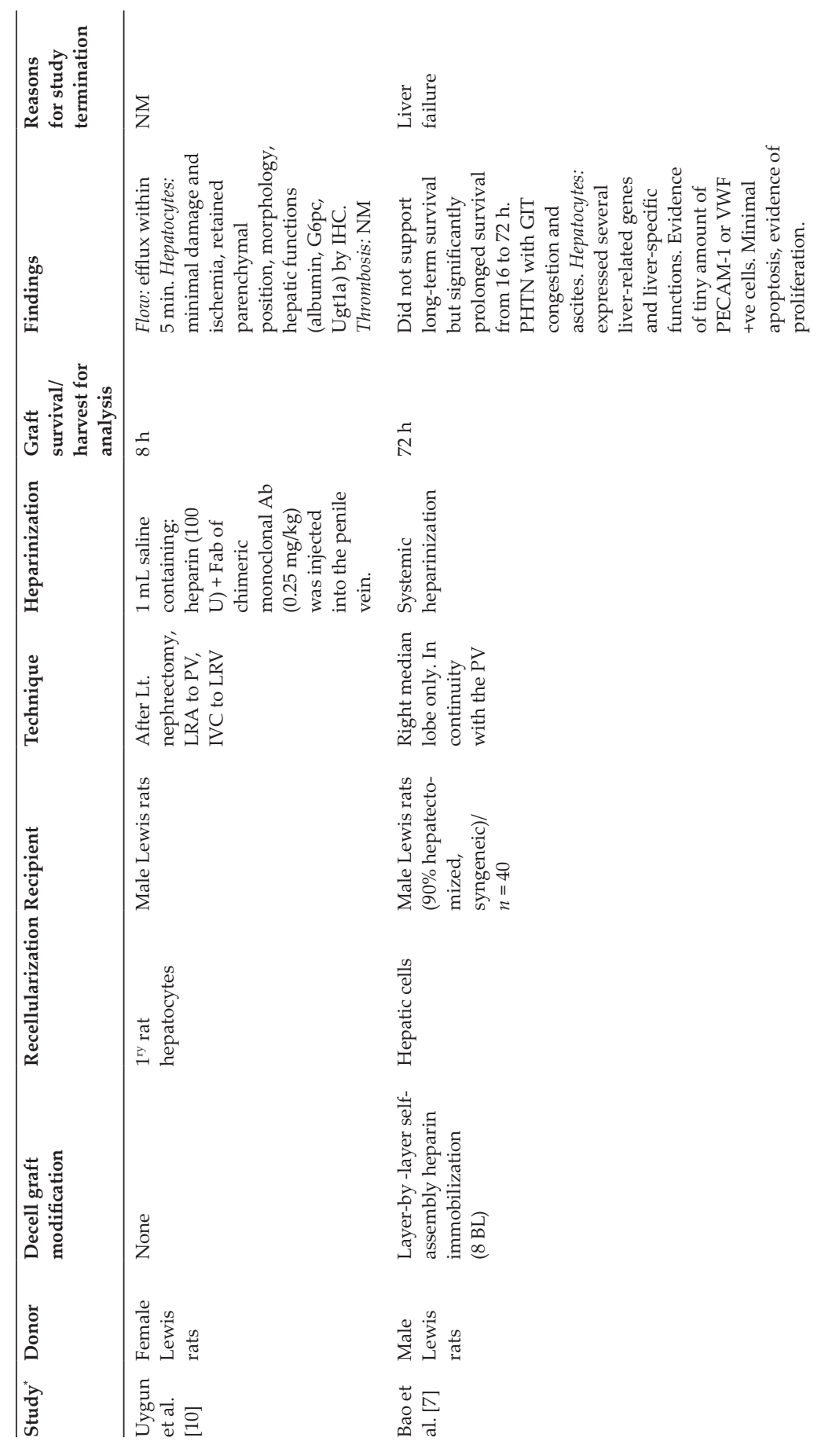




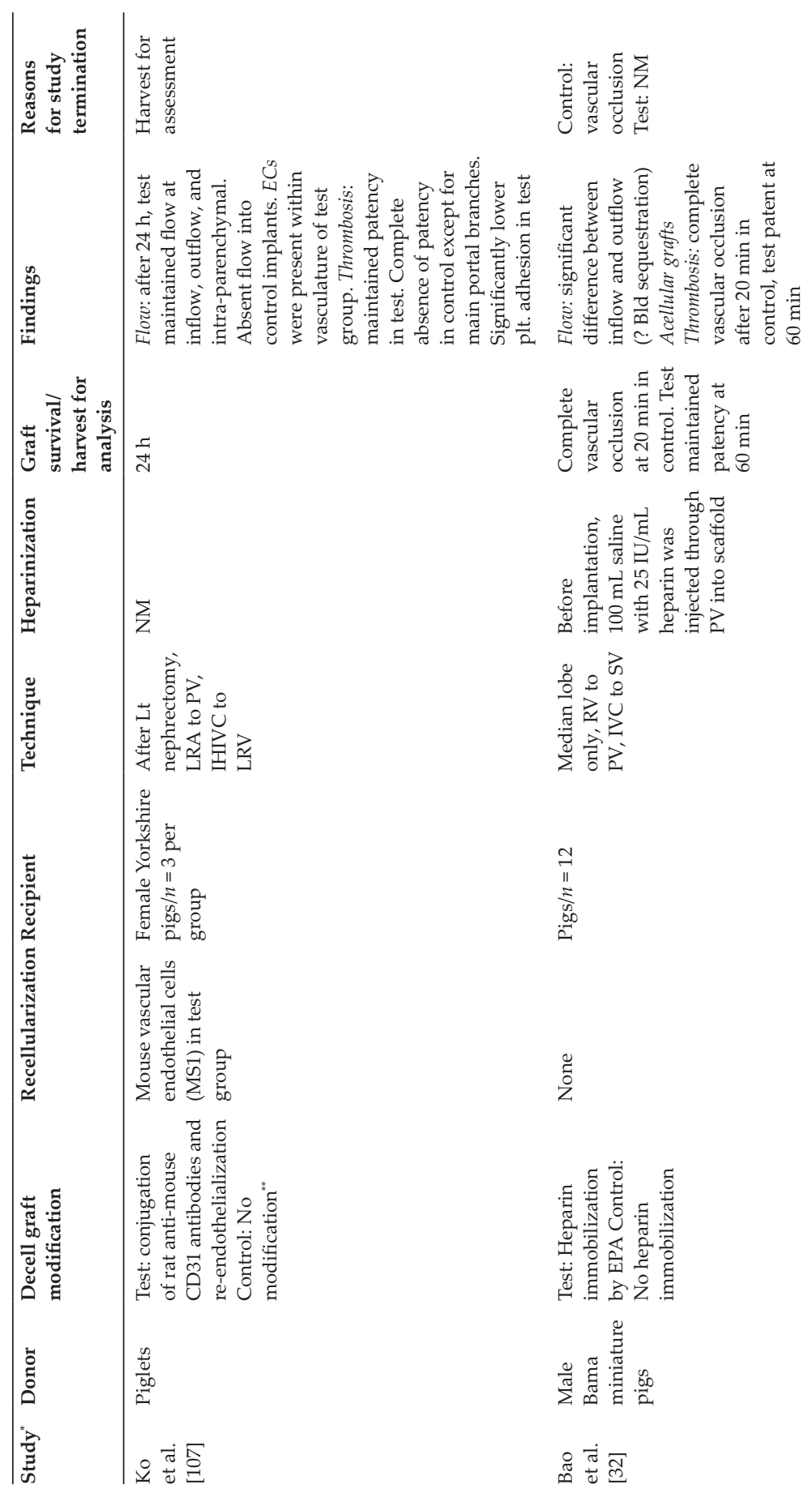




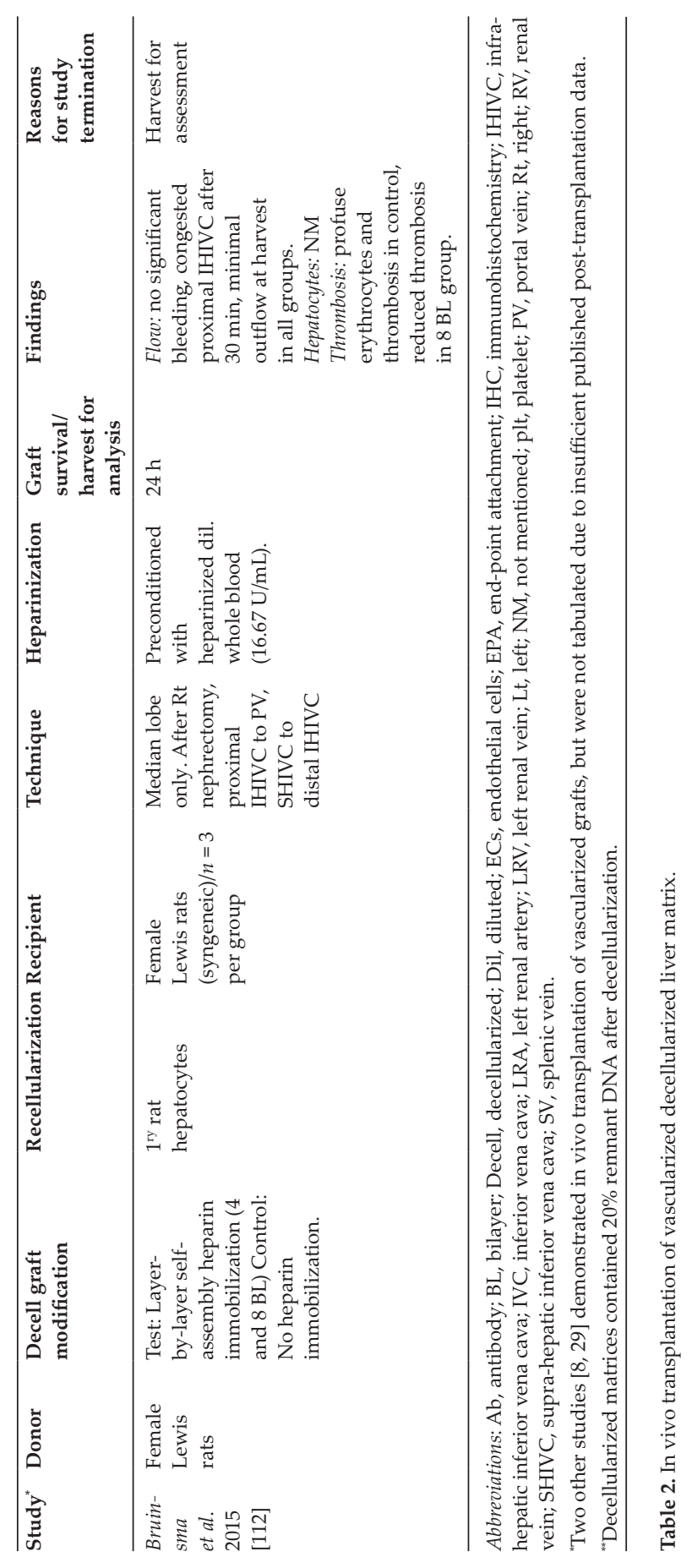


liver, porcine liver possesses several significant advantages. These include size/weight matching, animal availability, the ability to define the breeding and environmental exposure, and the availability of established techniques for genetic modification which can be combined with the decellularization protocols.

Standardization: standardization is a requirement for both the decellularization and sterilization protocols. Studies of whole-liver decellularization show great technical variability. A trial to define the optimal characterization cutoffs for all ECM constituents based on the in vivo outcomes seems futile at this stage. In a study by Mattei et al. ([37], preprint), the authors showed variation in decellularization efficiency of human immersion/agitation DLM from five donors despite using the same protocol. However, this observation was not highlighted in human perfusion DLM, where standard deviation for DNA content was $5.82 \mathrm{ng} / \mathrm{mg}$ [16]. Several studies described a relatively fast decellularization protocols for porcine [18], rabbit [9], and rat livers [25]. The utilization of automated perfusion systems, however, renders the duration of decellularization process a less important parameter, as opposed to the duration required for recellularization and ex vivo prepping.

Multi-organ decellularization: Park et al. [114] reported a technique for simultaneous multiorgan decellularization in rat through carotid artery and portal vein catheters. The process resulted in efficient decellularization of heart, liver, kidney, and other organs (e.g., stomach, intestine, etc.). This approach can prove practical for large-scale production of decellularized whole organs. On the other hand, the simultaneous decellularization of multiple whole livers using a multi-channel perfusion system is also possible.

Storage/preservation: cryopreservation and lyophilization are the techniques commonly advocated for the preservation of ECM products. Two techniques for cryopreservation include freezing and vitrification; hindering ice-crystal formation is the main challenge in both of them [108]. Washing of cryo-preservative agents is necessary after storage before proceeding with reseeding and implantation for cytotoxic concerns [108]. Poornejad et al. [115] studied the effects of freezing/thawing as a preservation technique for porcine whole-kidney decellularized scaffolds, without using cryoprotectants. Freezing/thawing did not affect the elastic modulus or adversely affect recellularization. However, this resulted in a decreased arterial pressure (as a measure of structural integrity) by a factor of 4 and caused partial damage of collagen and elastic fibers. The preservation of recellularized wDLMs remains unexplored.

Food and Drug Administration (FDA): eventually, decellularized/recellularized whole livers will have to meet FDA regulations in a similar fashion to commercially available decellularized human dermal grafts (e.g., AlloDerm ${ }^{\odot}$, Lifecell $^{\odot}$, etc.) for both the pre-market and post-market prerequisites. As a regenerative medicine technology, recellularized scaffolds fall in the "combination product" category which involves products that combine two or more regulated components that are produced as a single entity. Engineered whole livers can obtain market approval as either a "biologic" or "medical device" based on the mechanism of action [69].

Market potential: artificial organ market estimates consistently show enormous potentials due to the expanding transplantation demands. In 2014, a report by a US-based market research and consulting company estimated that the global artificial organ and bionics market can 
reach USD 38.75 billion by 2020. Artificial kidney took the lead in 2013 by global market of USD 12.21 billion. Interestingly, the artificial liver category was regarded as the most rapidly growing segment from 2014 to 2020 [116].

\section{Surgeon's perspective}

Engineered whole organs are considered a very attractive solution for organ-shortage crisis. It is thus important to elaborate on some fundamental parameters related to clinical application. Liver transplantation is indicated for a wide range of pathologies. Although indication-specific differences exist concerning the surgical technique and perioperative care, the graft-functional requirements are basically similar. Taking into consideration the variable approaches investigated for WLD, it can be noted that not all approaches are suitable for the different indications of liver transplantation. For example, a fully functioning liver graft will be required for fulminant liver failure, ex vivo liver-support device, end-stage liver failure, and malignancies. For these indications, lengthy ex vivo conditioning and in vivo proliferation approaches may not thus be suitable. Long ex vivo conditioning can be, however, acceptable in patients with biliary atresia, congenital absence of portal vein, or cirrhotic patients with lower model for end-stage liver disease (MELD)/pediatric end-stage liver disease (PELD) scores. In vivo proliferation can only be applied where auxiliary transplantation is feasible. Another example is in vivo decellularization; reasonably, this approach will not be suitable when an architectural abnormality is present as in the case of cirrhosis or biliary atresia, or in malignant conditions.

Implications of functional cell mass were discussed in Section 6. Problems related to the size of the graft can be anticipated in pediatric recipients, as a larger graft volume, in comparison to allografts, will be required to attain the required critical cell mass based on the current seeding densities. A smaller cell mass may be required in case of bridging, metabolic liver conditions, and acute liver failure. Feasibility of surgical implantation in different animal models was verified by the previously discussed in vivo experiments. PV anastomosis was feasible even without recellularization [32]. However, these studies did not tackle arterial or biliary anastomosis. In vivo studies that can accurately define the transplantation requirements are still lagging. Finally, the ability to produce an engineered liver that can be transplanted without the need of immunosuppression is highly desired. Still, the engineering of a liver graft from xenogeneic source that requires standard immunosuppression regimens similar to allograft will be a breakthrough, especially with the ever-increasing advances in the field of immune-suppressive medications.

\section{Conclusions}

In this chapter, an effort was made to put all the parameters of the WLD approach on display. This was done in order to link the different steps of the process together, to provide an overall insight of the approach progress and deficiencies, and to highlight the gaps in the published literature. It is undeniable that the understanding of the perfusion 
decellularization and the determinant factors of wDLM potentials has evidently evolved since the introduction of the concept in 2002. This understanding greatly challenged the simplicity of the approach viewed originally as crude borrowing of natural ECM platform and demanded for delicate appreciation of the ECM effectors. The simplicity of borrowing the human cell machinery and the patient-specific-tailoring concept on the other hand still lends the approach a very attractive edge among the other approaches of xenotransplantation. A higher appreciation of cell mechano-sensitivity, hepatic zonation, intra-wDLM flow dynamics, spatial cell-seeding organization, critical cell mass, and substrate modification can be easily noted in the recent publications. The WLD experimentation in a parallel, instead of sequential, fashion offered great insights and allowed a degree of feedback-based modifications. An awareness of the advances in the non-hepatic whole-organ decellularization trials, the other approaches for xenotransplantation, and material modification science is encouraged when dealing with WLD, together with accurate outlining of the clinical target. Therefore, a collaborative teamwork is necessary to offer complementary envisions. Finally, the suboptimal recellularization, intra-bioreactor cell function, and failures with in vivo long-term graft survival highlight deficiencies with two of the three main previously suggested players, namely the intrinsic DLM potentials and vascular competency. The third player (immune/host responses) remains largely unexplored for wDLM. The need for robust stepwise optimization is clear.

\section{Author details}

Ibrahim Fathi ${ }^{1,2 *}$ and Ahmed Eltawila ${ }^{3}$

*Address all correspondence to: I_Fathy201010@alexmed.edu.eg

1 Department of Surgery, Faculty of Medicine, University of Alexandria, Alexandria, Egypt

2 Division of Transplantation and Regenerative Medicine, Tohoku University School of Medicine, Tohoku, Japan

3 Department of Materials Science, Institute of Graduate Studies and Research, University of Alexandria, Alexandria, Egypt

\section{References}

[1] Atala A. Methods and compositions for organ decellularization. US Patent; US 6376244 B1, 2002

[2] Matthiesen TS, Ott HC, Goh S-K, Taylor DA. Large solid organ perfusion decellularization-A start for human-sized tissue scaffolds? Circulation. 2007;116(Suppl 16):II_103

[3] Gilbert TW, Sellaro TL, Badylak SF. Decellularization of tissues and organs. Biomaterials. 2006;27(19):3675-3683. DOI: 10.1016/j.biomaterials.2006.02.014 
[4] Crapo PM, Gilbert TW, Badylak SF. An overview of tissue and whole organ decellularization processes. Biomaterials. 2011;32(12):3233-3243. DOI: 10.1016/j.biomaterials. 2011.01.057

[5] He M, Callanan A. Comparison of methods for whole-organ decellularization in tissue engineering of bioartificial organs. Tissue Engineering. Part B: Reviews. 2012;19(3):194208. DOI: $10.1089 /$ ten.teb.2012.0340

[6] Wang Y, Nicolas C, Chen H, Ross J, De Lorenzo S, Nyberg S. Recent advances in decellularization and recellularization for tissue-engineered liver grafts. Cells Tissues Organs. 2017;203:203-214. DOI: 10.1159/000452761

[7] Bao J, Shi Y, Sun H, Yin X, Yang R, Li L, Chen X, Bu H. Construction of a portal implantable functional tissue-engineered liver using perfusion-decellularized matrix and hepatocytes in rats. Cell Transplantation. 2011;20(5):753-766. DOI: 10.3727/096368910X536572

[8] Barakat O, Abbasi S, Rodriguez G, Rios J, Wood RP, Ozaki C, Holley LS, Gauthier PK. Use of decellularized porcine liver for engineering humanized liver organ. Journal of Surgical Research. 2012;173(1):e11-e25. DOI: 10.1016/j.jss.2011.09.033

[9] Fathi I, Elhammady H, Sakr M, Nabawi A, Marei M. Rapid hepatic perfusion decellularization: Technique and critique. Xenotransplantation. 2015 Nov-Dec;22(6):451-457. PubMed PMID: 26669725. Epub 2015/12/17. eng. DOI: 10.1111/xen.12212

[10] Uygun BE, Soto-Gutierrez A, Yagi H, Izamis M-L, Guzzardi MA, Shulman C, Milwid J, Kobayashi N, Tilles A, Berthiaume F. Organ reengineering through development of a transplantable recellularized liver graft using decellularized liver matrix. Nature Medicine. 2010;16(7):814-820. DOI: 10.1038/nm.2170

[11] Yagi H, Fukumitsu K, Fukuda K, Kitago M, Shinoda M, Obara H, Itano O, Kawachi S, Tanabe M, Coudriet GM. Human-scale whole-organ bioengineering for liver transplantation: A regenerative medicine approach. Cell Transplantation. 2013;22(2):231-242. DOI:10.3727/096368912X654939

[12] White LJ, Taylor AJ, Faulk DM, Keane TJ, Saldin LT, Reing JE, Swinehart IT, Turner NJ, Ratner BD, Badylak SF. The impact of detergents on the tissue decellularization process: A ToF-SIMS study. Acta Biomaterialia. 2017;50:207-219. DOI: 10.1016/j.actbio.2016.12.033

[13] Jiang W-C, Cheng Y-H, Yen M-H, Chang Y, Yang VW, Lee OK. Cryo-chemical decellularization of the whole liver for mesenchymal stem cells-based functional hepatic tissue engineering. Biomaterials. 2014;35(11):3607-3617. DOI: 10.1016/j.biomaterials.2014.01.024

[14] Kajbafzadeh A-M, Javan-Farazmand N, Monajemzadeh M, Baghayee A. Determining the optimal decellularization and sterilization protocol for preparing a tissue scaffold of a human-sized liver tissue. Tissue Engineering. Part C: Methods. 2013;19(8):642-651. DOI: 10.1089/ten.tec.2012.0334

[15] Gessner RC, Hanson AD, Feingold S, Cashion AT, Corcimaru A, Wu BT, Mullins CR, Aylward SR, Reid LM, Dayton PA. Functional ultrasound imaging for assessment 
of extracellular matrix scaffolds used for liver organoid formation. Biomaterials. 2013;34(37):9341-9351. DOI: 10.1016/j.biomaterials.2013.08.033

[16] Mazza G, Rombouts K, Hall AR, Urbani L, Luong TV, Al-Akkad W, Longato L, Brown D, Maghsoudlou P, Dhillon AP. Decellularized human liver as a natural 3D-scaffold for liver bioengineering and transplantation. Scientific Reports. 2015;5:13079. DOI: 10.1038/ srep13079

[17] Bourgine PE, Gaudiello E, Pippenger B, Jaquiery C, Klein T, Pigeot S, Todorov A, Feliciano S, Banfi A, Martin I. Engineered extracellular matrices as biomaterials of tunable composition and function. Advanced Functional Materials. 2017;27(7). DOI: 10.1002/ adfm.201605486

[18] Struecker B, Butter A, Hillebrandt K, Polenz D, Reutzel-Selke A, Tang P, Lippert S, Leder A, Rohn S, Geisel D. Improved rat liver decellularization by arterial perfusion under oscillating pressure conditions. Journal of Tissue Engineering and Regenerative Medicine. 2017;11(2):531-541. DOI: 10.1002/term.1948

[19] Pan J, Yan S, Gao J-j, Wang Y-y, Lu Z-j, Cui C-w, Zhang Y-h, Wang Y, Meng X-q, Zhou L. In-vivo organ engineering: Perfusion of hepatocytes in a single liver lobe scaffold of living rats. The International Journal of Biochemistry \& Cell Biology. 2016;80:124-131. DOI: 10.1016/j.biocel.2016.10.003

[20] He M, Callanan A, Lagaras K, Steele J, Stevens M. Optimization of SDS exposure on preservation of ECM characteristics in whole organ decellularization of rat kidneys. Journal of Biomedical Materials Research Part B: Applied Biomaterials. 2016. DOI: 10.1002/jbm.b.33668

[21] Sabetkish S, Kajbafzadeh AM, Sabetkish N, Khorramirouz R, Akbarzadeh A, Seyedian SL, Pasalar P, Orangian S, Beigi RSH, Aryan Z. Whole-organ tissue engineering: Decellularization and recellularization of three-dimensional matrix liver scaffolds. Journal of Biomedical Materials Research Part A. 2015;103(4):1498-1508. DOI: 10.1002/ jbm.a.35291

[22] Maghsoudlou P, Georgiades F, Smith H, Milan A, Shangaris P, Urbani L, Loukogeorgakis SP, Lombardi B, Mazza G, Hagen C. Optimization of liver decellularization maintains extracellular matrix micro-architecture and composition predisposing to effective cell seeding. PLoS One. 2016;11(5):e0155324. DOI: 10.1371/journal.pone.0155324

[23] Shirakigawa N, Ijima H, Takei T. Decellularized liver as a practical scaffold with a vascular network template for liver tissue engineering. Journal of Bioscience and Bioengineering. 2012;114(5):546-551. DOI: 10.1016/j.jbiosc.2012.05.022

[24] Mattei G, Di Patria V, Tirella A, Alaimo A, Elia G, Corti A, Paolicchi A, Ahluwalia A. Mechanostructure and composition of highly reproducible decellularized liver matrices. Acta Biomaterialia. 2014;10(2):875-882. DOI: 10.1016/j.actbio.2013.10.023 
[25] De Kock J, Ceelen L, De Spiegelaere W, Casteleyn C, Claes P, Vanhaecke T, Rogiers V. Simple and quick method for whole-liver decellularization: A novel in vitro three-dimensional bioengineering tool? Archives of Toxicology. 2011;85(6):607-612. DOI: 10.1007/s00204-011-0706-1

[26] Shupe T, Williams M, Brown A, Willenberg B, Petersen BE. Method for the decellularization of intact rat liver. Organogenesis. 2010;6(2):134-136. DOI: 10.4161/org.6.2.11546

[27] Soto-Gutierrez A, Zhang L, Medberry C, Fukumitsu K, Faulk D, Jiang H, Reing J, Gramignoli $\mathrm{R}$, Komori J, Ross M. A whole-organ regenerative medicine approach for liver replacement. Tissue Engineering. Part C: Methods. 2011;17(6):677-686. DOI: 10.1089/ten.tec.2010.0698

[28] Baptista PM, Orlando G, Mirmalek-Sani S-H, Siddiqui M, Atala A, Soker S, editors. Whole organ decellularization-a tool for bioscaffold fabrication and organ bioengineering. Conference Proceedings - IEEE Engineering in Medicine and Biology Society. 2009;2009:6526-6529. DOI: 10.1109/IEMBS.2009.5333145

[29] Baptista PM, Siddiqui MM, Lozier G, Rodriguez SR, Atala A, Soker S. The use of whole organ decellularization for the generation of a vascularized liver organoid. Hepatology. 2011;53(2):604-617. DOI: 10.1002/hep.24067

[30] Federico S, Ayelen R, Romina C, Nelly BA, Adrian NG, Alicia SN, Paula CM, editors. Obtaining liver tridimensional scaffold through the decellularization of rabbit whole liver in 24 hours. Journal of Physics: Conference Series. 2016;705(1):012011. DOI: 10.1088/1742-6596/705/1/012011

[31] Nari GA, Cid M, Comin R, Reyna L, Juri G, Taborda R, Salvatierra NA. Preparation of a three-dimensional extracellular matrix by decellularization of rabbit livers. Revista Espanola de Enfermedades Digestivas: Organo Oficial de la Sociedad Espanola de Patologia Digestiva. 2013;105(3):138-143. DOI: 10.4321/S1130-01082013000300004

[32] Bao J, Wu Q, Sun J, Zhou Y, Wang Y, Jiang X, Li L, Shi Y, Bu H. Hemocompatibility improvement of perfusion-decellularized clinical-scale liver scaffold through heparin immobilization. Scientific Reports. 2015;5:10756. DOI: 10.1038/srep10756

[33] Lang R, Stern MM, Smith L, Liu Y, Bharadwaj S, Liu G, Baptista PM, Bergman CR, Soker S, Yoo JJ, Atala A, Zhang Y. Three-dimensional culture of hepatocytes on porcine liver tissue-derived extracellular matrix. Biomaterials. 2011 Oct;32(29):7042-7052

[34] Struecker B, Hillebrandt KH, Voitl R, Butter A, Schmuck RB, Reutzel-Selke A, Geisel D, Joehrens K, Pickerodt PA, Raschzok N, Puhl G, Neuhaus P, Pratschke J, Sauer IM. Porcine liver decellularization under oscillating pressure conditions: A technical refinement to improve the homogeneity of the decellularization process. Tissue Engineering Part C Methods. 2015 Mar;21(3):303-313

[35] Zhang Z, Bédard E, Luo Y, Wang H, Deng S, Kelvin D, Zhong R. Animal models in xenotransplantation. Expert Opinion on Investigational Drugs. 2000;9(9):2051-2068. DOI: 10.1517/13543784.9.9.2051 
[36] Wu Q, Bao J, Zhou Y-j, Wang Y-j, Du Z-g, Shi Y-j, Li L, Bu H. Optimizing perfusion-decellularization methods of porcine livers for clinical-scale whole-organ bioengineering. BioMed Research International. 2015;2015:785474. DOI: 10.1155/2015/785474

[37] Mattei G, Magliaro C, Pirone A, Ahluwalia A. Decellularised human liver is too heterogeneous for designing a generic ECM-mimic hepatic scaffold. Pre-print. bioRxiv 2016:073270. DOI: 10.1101/073270

[38] Badylak SF, Freytes DO, Gilbert TW. Extracellular matrix as a biological scaffold material: Structure and function. Acta Biomaterialia. 2009;5(1):1-13. DOI: 10.1016/j. actbio.2008.09.013

[39] Martinez-HernandezA, Amenta P. The hepatic extracellular matrix. II. Ontogenesis, regeneration and cirrhosis. Virchows Archiv A, Pathological Anatomy and Histopathology. 1992;423(2):77-84. DOI: 10.1007/BF01606580

[40] Martinez-Hernandez A, Amenta PS. The hepatic extracellular matrix. I. Components and distribution in normal liver. Virchows Arch A, Pathological Anatomy and Histopathology. 1993;423(1):1-11

[41] Nagata S, Hanayama R, Kawane K. Autoimmunity and the clearance of dead cells. Cell. 2010;140(5):619-630. DOI: 10.1016/j.cell.2010.02.014

[42] Zhang Q, Raoof M, Chen Y, Sumi Y, Sursal T, Junger W, Brohi K, Itagaki K, Hauser CJ. Circulating mitochondrial DAMPs cause inflammatory responses to injury. Nature. 2010;464(7285):104-107. DOI: 10.1038/nature08780

[43] San Antonio JD, Iozzo RV. Glycosaminoglycans: Structure and biological functions. In: eLS. Chichester: John Wiley \& Sons Ltd.; 2001. DOI: 10.1038/npg.els.0000704LS

[44] Wang Y, Cui CB, Yamauchi M, Miguez P, Roach M, Malavarca R, Costello MJ, Cardinale $\mathrm{V}$, Wauthier E, Barbier C. Lineage restriction of human hepatic stem cells to mature fates is made efficient by tissue-specific biomatrix scaffolds. Hepatology. 2011;53(1):293-305. DOI: 10.1002/hep.24012

[45] Caralt M, Uzarski JS, Iacob S, Obergfell KP, Berg N, Bijonowski BM, Kiefer KM, Ward $\mathrm{HH}$, Wandinger-Ness A, Miller WM. Optimization and critical evaluation of decellularization strategies to develop renal extracellular matrix scaffolds as biological templates for organ engineering and transplantation. American Journal of Transplantation. 2015;15(1):64-75. DOI: 10.1111/ajt.12999

[46] Badylak SF, Gilbert TW, editors. Immune response to biologic scaffold materials. Seminars in Immunology. 2008;20(2):109-116. DOI: 10.1016/j.smim.2007.11.003

[47] Engler AJ, Sen S, Sweeney HL, Discher DE. Matrix elasticity directs stem cell lineage specification. Cell. 2006;126(4):677-689. DOI: 10.1016/j.cell.2006.06.044

[48] Lozoya OA, Wauthier E, Turner RA, Barbier C, Prestwich GD, Guilak F, Superfine R, Lubkin SR, Reid LM. Regulation of hepatic stem/progenitor phenotype by microenvironment 
stiffness in hydrogel models of the human liver stem cell niche. Biomaterials. 2011;32(30): 7389-7402. DOI: 10.1016/j.biomaterials.2011.06.042

[49] Dash A, Simmers MB, Deering TG, Berry DJ, Feaver RE, Hastings NE, Pruett TL, LeCluyse EL, Blackman BR, Wamhoff BR. Hemodynamic flow improves rat hepatocyte morphology, function, and metabolic activity in vitro. American Journal of PhysiologyCell Physiology. 2013;304(11):C1053-C1063. DOI: 10.1152/ajpcell.00331.2012

[50] Miki T, Ring A, Gerlach J. Hepatic differentiation of human embryonic stem cells is promoted by three-dimensional dynamic perfusion culture conditions. Tissue Engineering. Part C: Methods. 2011;17(5):557-568. DOI: 10.1089/ten.tec.2010.0437

[51] Schmelzer E, Triolo F, Turner ME, Thompson RL, Zeilinger K, Reid LM, Gridelli B, Gerlach JC. Three-dimensional perfusion bioreactor culture supports differentiation of human fetal liver cells. Tissue Engineering. Part A. 2010;16(6):2007-2016. DOI: 10.1089/ ten.tea.2009.0569

[52] Hsu W-M, Carraro A, Kulig KM, Miller ML, Kaazempur-Mofrad M, Weinberg E, Entabi F, Albadawi H, Watkins MT, Borenstein JT. Liver-assist device with a microfluidicsbased vascular bed in an animal model. Annals of Surgery. 2010;252(2):351-357. DOI: 10.1097/SLA.0b013e3181e982ba

[53] Schön MR, Kollmar O, Wolf S, Schrem H, Matthes M, Akkoc N, Schnoy NC, Neuhaus P. Liver transplantation after organ preservation with normothermic extracorporeal perfusion. Annals of Surgery. 2001;233(1):114-123. DOI: 10.1097/00000658-200101000-00017

[54] Evans DW, Moran EC, Baptista PM, Soker S, Sparks JL. Scale-dependent mechanical properties of native and decellularized liver tissue. Biomechanics and Modeling in Mechanobiology. 2013 Jun;12(3):569-580. DOI: 10.1007/s10237-012-0426-3

[55] Klaas M, Kangur T, Viil J, Mäemets-Allas K, Minajeva A, Vadi K, Antsov M, Lapidus N, Järvekülg $\mathrm{M}$, Jaks V. The alterations in the extracellular matrix composition guide the repair of damaged liver tissue. Scientific Reports. 2016;6:27398. DOI: 10.1038/srep27398

[56] Geerts S, Ozer S, Jaramillo M, Yarmush ML, Uygun BE. Nondestructive methods for monitoring cell removal during rat liver decellularization. Tissue Engineering. Part C: Methods. 2016;22(7):671-678. DOI: 10.1089/ten.tec.2015.0571

[57] Hagen CK, Maghsoudlou P, Totonelli G, Diemoz PC, Endrizzi M, Rigon L, Menk R-H, Arfelli F, Dreossi D, Brun E. High contrast microstructural visualization of natural acellular matrices by means of phase-based X-ray tomography. Scientific Reports. 2015;5:18156. DOI: $10.1038 /$ srep18156

[58] Gilbert TW, Stewart-Akers AM, Simmons-Byrd A, Badylak SF. Degradation and remodeling of small intestinal submucosa in canine Achilles tendon repair. Journal of Bone and Joint Surgery. 2007;89(3):621-630. DOI: 10.2106/00004623-200703000-00020

[59] Zhou P, Lessa N, Estrada DC, Severson EB, Lingala S, Zern MA, Nolta JA, Wu J. Decellularized liver matrix as a carrier for the transplantation of human fetal and 
primary hepatocytes in mice. Liver Transplantation. 2011;17(4):418-427. DOI: 10.1002/ 1t. 22270

[60] Wells RG. Cellular sources of extracellular matrix in hepatic fibrosis. Clinics in Liver Disease. 2008;12(4):759-768. DOI: 10.1016/j.cld.2008.07.008

[61] Arnaud A, Fontana L, Angulo AJ, Gil Á, López-Pedrosa JM. Proliferation, functionality, and extracellular matrix production of hepatocytes and a liver stellate cell line: A comparison between single cultures and cocultures. Digestive Diseases and Sciences. 2003;48(7):1406-1413. DOI: 10.1023/A:1024192100775

[62] Caralt M, Velasco E, Lanas A, Baptista PM. Liver bioengineering: From the stage of liver decellularized matrix to the multiple cellular actors and bioreactor special effects. Organogenesis. 2014;10(2):250-259. DOI: 10.4161/org.29892

[63] McClelland R, Wauthier E, Uronis J, Reid L. Gradients in the liver's extracellular matrix chemistry from periportal to pericentral zones: Influence on human hepatic progenitors. Tissue Engineering. Part A. 2008;14(1):59-70. DOI: 10.1089/ten.a.2007.0058

[64] Susick R, Moss N, Kubota H, Lecluyse E, Hamilton G, Luntz T, Ludlow J, Fair J, Gerber D, Bergstrand K. Hepatic progenitors and strategies for liver cell therapies. Annals of the New York Academy of Sciences. 2001;944(1):398-419. DOI: 10.1111/j.1749-6632.2001.tb03851.x

[65] Reid LM, Fiorino AS, Sigal SH, Brill S, Holst PA. Extracellular matrix gradients in the space of Disse: Relevance to liver biology. Hepatology. 1992;15(6):1198-1203. DOI: 10.1002/hep.1840150635

[66] Kietzmann T. Metabolic zonation of the liver: The oxygen gradient revisited. Redox Biology. 2017. DOI: 10.1016/j.redox.2017.01.012

[67] Beresford W, Henninger J. A tabular comparative histology of the liver. Archivum Histologicum Japonicum. 1986;49(3):267-281. DOI: 10.1679/aohc.49.267

[68] Wang X, Cui J, Zhang BQ, Zhang H, Bi Y, Kang Q, Wang N, Bie P, Yang Z, Wang H. Decellularized liver scaffolds effectively support the proliferation and differentiation of mouse fetal hepatic progenitors. Journal of Biomedical Materials Research Part A. 2014;102(4):1017-1025. DOI: 10.1002/jbm.a.34764

[69] Scarritt ME, Pashos NC, Bunnell BA. A review of cellularization strategies for tissue engineering of whole organs. Frontiers in Bioengineering and Biotechnology. 2015;3:43. DOI: $10.3389 /$ fbioe. 2015.00043

[70] Hu X, Yang T, Li C, Zhang L, Li M, Huang W, Zhou P, editors. Human fetal hepatocyte line, L-02, exhibits good liver function in vitro and in an acute liver failure model. Transplantation Proceedings. 2013;45(2):695-700. DOI: 10.1016/j.transproceed.2012.09.121

[71] Palakkan AA, Hay DC, TV K, Ross JA. Liver tissue engineering and cell sources: Issues and challenges. Liver International. 2013;33(5):666-676. DOI: 10.1111/liv.12134

[72] Walldorf J, Aurich H, Cai H, Runge D, Christ B, Strom S, Fleig W. Expanding hepatocytes in vitro before cell transplantation: Donor age-dependent proliferative capacity of 
cultured human hepatocytes. Scandinavian Journal of Gastroenterology. 2004;39(6):584593. DOI: $10.1080 / 00365520410005586$

[73] Satyananda V, Hara H, Ezzelarab MB, Phelps C, Ayares D, Cooper DK. New concepts of immune modulation in xenotransplantation. Transplantation. 2013;96(11):937. DOI: 10.1097/TP.0b013e31829bbcb2

[74] Mirmalek-SaniS-H, Sullivan DC, Zimmerman C, Shupe TD, Petersen BE. Immunogenicity of decellularized porcine liver for bioengineered hepatic tissue. American Journal of Pathology. 2013;183(2):558-565. DOI: 10.1016/j.ajpath.2013.05.002

[75] Hassanein W, Uluer MC, Langford J, Woodall JD, Cimeno A, Dhru U, Werdesheim A, Harrison J, Rivera-Pratt C, Klepfer S. Recellularization via the bile duct supports functional allogenic and xenogenic cell growth on a decellularized rat liver scaffold. Organogenesis. 2017;13(1):16-27. DOI: 10.1080/15476278.2016.1276146

[76] Ogiso S, Yasuchika K, Fukumitsu K, Ishii T, Kojima H, Miyauchi Y, Yamaoka R, Komori J, Katayama H, Kawai T. Efficient recellularisation of decellularised whole-liver grafts using biliary tree and foetal hepatocytes. Scientific Reports. 2016;6:35887. DOI: 10.1038/ srep35887

[77] Gupta S, Rajvanshi P, Sokhi R, Slehria S, Yam A, Kerr A, Novikoff PM. Entry and integration of transplanted hepatocytes in rat liver plates occur by disruption of hepatic sinusoidal endothelium. Hepatology. 1999;29(2):509-519. DOI: 10.1002/hep.510290213

[78] Kholodenko IV, Yarygin KN. Cellular mechanisms of liver regeneration and cell-based therapies of liver diseases. BioMed Research International. 2017;2017. DOI: 10.1155/ $2017 / 8910821$

[79] Sohlenius-Sternbeck A-K. Determination of the hepatocellularity number for human, $\mathrm{dog}$, rabbit, rat and mouse livers from protein concentration measurements. Toxicology in Vitro. 2006;20(8):1582-1586. DOI: 10.1016/j.tiv.2006.06.003

[80] Budd G. Liver stereology. In: Motta P, Didio L, editors. Basic and Clinical Hepatology. Vol. 2. Springer Science \& Business Media, Netherlands; 2012. p. 111

[81] Xu Q, Sun X, Qiu Y, Zhang H, Ding Y. The optimal hepatocyte density for a hollow-fiber bioartificial liver. Annals of Clinical \& Laboratory Science. 2004;34(1):87-93

[82] Dvir-Ginzberg M, Gamlieli-Bonshtein I, Agbaria R, Cohen S. Liver tissue engineering within alginate scaffolds: Effects of cell-seeding density on hepatocyte viability, morphology, and function. Tissue Engineering. 2003;9(4):757-766. DOI: 10.1089/ 107632703768247430

[83] McPherson T, Liang H, Record R, Badylak S. Gal $\alpha(1,3)$ Gal epitope in porcine small intestinal submucosa. Tissue Engineering. 2000;6(3):233-239. DOI: 10.1089/10763270050044416

[84] Stone KR, Ayala G, Goldstein J, Hurst R, Walgenbach A, Galili U. Porcine cartilage transplants in the cynomolgus monkey: III. Transplantation of $\alpha$-galactosidase-treated porcine cartilage.Transplantation.1998;65(12):1577-1583.DOI:10.1097/00007890-199806270-00007 
[85] Hamadeh R, Jarvis G, Galili U, Mandrell R, Zhou P, Griffiss J. Human natural anti-Gal IgG regulates alternative complement pathway activation on bacterial surfaces. Journal of Clinical Investigation. 1992;89(4):1223. DOI: 10.1172/JCI115706

[86] Yu PB, Holzknecht ZE, Bruno D, Parker W, Platt JL. Modulation of natural IgM binding and complement activation by natural IgG antibodies: A role for IgG anti-Gal alpha13Gal antibodies. Journal of Immunology. 1996;157(11):5163-5168

[87] Brljggemann M, Williams GT, Bindon CI, Clark MR, Walker MR. Comparison of the effector functions of human immunoglobulins using a matched set of chimeric antibodies. Journal of Experimental Medicine. 1987;166(5):1351-1361

[88] Galili U. Interaction of the natural anti-Gal antibody with $\alpha$-galactosyl epitopes: A major obstacle for xenotransplantation in humans. Immunology Today. 1993;14(10):480-482. DOI: 10.1016/0167-5699(93)90261-I

[89] Raeder RH, Badylak SF, Sheehan C, Kallakury B, Metzger DW. Natural anti-galactose $\alpha 1,3$ galactose antibodies delay, but do not prevent the acceptance of extracellular matrix xenografts. Transplant Immunology. 2002;10(1):15-24. DOI: 10.1016/S09663274(01)00044-2

[90] Derwin KA, Baker AR, Spragg RK, Leigh DR, Iannotti JP. Commercial extracellular matrix scaffolds for rotator cuff tendon repair. Journal of Bone and Joint Surgery (American). 2006;88(12):2665-2672. DOI: 10.2106/00004623-200612000-00014

[91] Zheng M, Chen J, Kirilak Y, Willers C, Xu J, Wood D. Porcine small intestine submucosa (SIS) is not an acellular collagenous matrix and contains porcine DNA: Possible implications in human implantation. Journal of Biomedical Materials Research Part B: Applied Biomaterials. 2005;73(1):61-67. DOI: 10.1002/jbm.b.30170

[92] Valentin JE, Badylak JS, McCabe GP, Badylak SF. Extracellular matrix bioscaffolds for orthopaedic applications. Journal of Bone and Joint Surgery (American). 2006;88(12):26732686. DOI: 10.2106/00004623-200612000-00015

[93] Kant CD, Akiyama Y, Tanaka K, Shea S, Connolly SE, Germana S, Winn HJ, LeGuern C, Tocco G, Benichou G. Primary vascularization of allografts governs their immunogenicity and susceptibility to tolerogenesis. Journal of Immunology. 2013;191(4):1948-1956. DOI: 10.4049/jimmunol.1202092

[94] Kwun J, Malarkannan S, Burlingham WJ, Knechtle SJ. Primary vascularization of the graft determines the immunodominance of murine minor $\mathrm{H}$ antigens during organ transplantation. The Journal of Immunology. 2011;187(8):3997-4006. DOI: 10.4049/ jimmunol.1003918

[95] Galili U, Macher BA, Buehler J, Shohet SB. Human natural anti-alpha-galactosyl IgG. II. The specific recognition of alpha (1----3)-linked galactose residues. Journal of Experimental Medicine. 1985;162(2):573-582. DOI: 10.1084/jem.162.2.573 
[96] Good A, Cooper D, Malcolm A, Ippolito R, Koren E, Neethling F, Ye Y, Zuhdi N, Lamontagne L. Identification of carbohydratestructures that bind human antiporcine antibodies: Implications for discordant xenografting in humans. Transplantation Proceedings; 1992;24:559-562

[97] Platt JL, Fischel RJ, Matas AJ, Reif SA, Bolman RM, Bach FH. Immunopathology of hyperacute xenograft rejection in a swine-to-primate model. Transplantation. 1991;52(2):214-220. DOI: 10.1097/00007890-199108000-00006

[98] Hossler FE, Douglas JE. Vascular corrosion casting: Review of advantages and limitations in the application of some simple quantitative methods. Microscopy and Microanalysis. 2001;7(03):253-264. DOI: 10.1017/S1431927601010261

[99] Esmon CT. Basic mechanisms and pathogenesis of venous thrombosis. Blood Reviews. 2009;23(5):225-229. DOI: 10.1016/j.blre.2009.07.002

[100] Robertson MJ, Dries-Devlin JL, Kren SM, Burchfield JS, Taylor DA. Optimizing recellularization of whole decellularized heart extracellular matrix. PLoS One. 2014;9(2):e90406. DOI: 10.1371/journal.pone.0090406

[101] Nagaoka Y, Yamada H, Kimura T, Kishida A, Fujisato T, Takakuda K. Reconstruction of small diameter arteries using decellularized vascular scaffolds. Journal of Medical and Dental Sciences. 2014;61(1):33-40. DOI: 10.11480/610105

[102] Wang XN, Chen CZ, Yang M, Gu YJ. Implantation of decellularized small-caliber vascular xenografts with and without surface heparin treatment. Artificial Organs. 2007;31(2):99-104. DOI: 10.1111/j.1525-1594.2007.00348.x

[103] Bajpai VK, Andreadis ST. Stem cell sources for vascular tissue engineering and regeneration. Tissue Engineering. Part B: Reviews. 2012;18(5):405-425. DOI: 10.1089/ten.teb.2011.0264

[104] Lozito TP, Kuo CK, Taboas JM, Tuan RS. Human mesenchymal stem cells express vascular cell phenotypes upon interaction with endothelial cell matrix. Journal of Cellular Biochemistry. 2009;107(4):714-722. DOI: 10.1002/jcb.22167

[105] Liu JY, Swartz DD, Peng HF, Gugino SF, Russell JA, Andreadis ST. Functional tissueengineered blood vessels from bone marrow progenitor cells. Cardiovascular Research. 2007;75(3):618-628. DOI: 10.1016/j.cardiores.2007.04.018

[106] Marra KG, Brayfield CA, Rubin JP. Adipose stem cell differentiation into smooth muscle cells.In: Gimble J, BunnellB, editors. Adipose-Derived Stem Cells: Methods and Protocols. Humana Press, New York; 2011.pp. 261-268. DOI: 10.1007/978-1-61737-960-4_19

[107] Ko IK, Peng L, Peloso A, Smith CJ, Dhal A, Deegan DB, Zimmerman C, Clouse C, Zhao $\mathrm{W}$, Shupe TD. Bioengineered transplantable porcine livers with re-endothelialized vasculature. Biomaterials. 2015;40:72-79. DOI: 10.1016/j.biomaterials.2014.11.027

[108] Seifu DG, Purnama A, Mequanint K, Mantovani D. Small-diameter vascular tissue engineering. Nature Reviews Cardiology. 2013;10(7):410-421. DOI: 10.1038/nrcardio.2013.77 
[109] Haugen G, Kiserud T, Godfrey K, Crozier S, Hanson M. Portal and umbilical venous blood supply to the liver in the human fetus near term. Ultrasound in Obstetrics \& Gynecology. 2004;24(6):599-605. DOI: 10.1002/uog.1744

[110] Nishii K, Reese G, Moran EC, Sparks JL. Multiscale computational model of fluid flow and matrix deformation in decellularized liver. Journal of the Mechanical Behavior of Biomedical Materials. 2016;57:201-214. DOI: 10.1016/j.jmbbm.2015.11.033

[111] Ren X, Tapias LF, Jank BJ, Mathisen DJ, Lanuti M, Ott HC. Ex vivo non-invasive assessment of cell viability and proliferation in bio-engineered whole organ constructs. Biomaterials. 2015;52:103-112. DOI: 10.1016/j.biomaterials.2015.01.061

[112] Bruinsma BG, Kim Y, Berendsen TA, Ozer S, Yarmush ML, Uygun BE. Layer-by-layer heparinization of decellularized liver matrices to reduce thrombogenicity of tissue engineered grafts. Journal of Clinical and Translational Research. 2015;1(1):pii: 04. DOI: 10.18053/jctres.201501.004

[113] Sarikaya A, Record R, Wu C-C, Tullius B, Badylak S, Ladisch M. Antimicrobial activity associated with extracellular matrices. Tissue Engineering. 2002;8(1):63-71. DOI: 10.1089/107632702753503063

[114] Park K, Woo H, editors. Systemic decellularization for multi-organ scaffolds in rats. Transplantation Proceedings. 2012;44(4):1151-1154.DOI:10.1016/j.transproceed.2012.03.017

[115] Poornejad N, Frost TS, Scott DR, Elton BB, Reynolds PR, Roeder BL, Cook AD. Freezing/ thawing without cryoprotectant damages native but not decellularized porcine renal tissue. Organogenesis. 2015;11(1):30-45. DOI: 10.1080/15476278.2015.1022009

[116] Global Artificial Organ and Bionics Market. United States: Grand View Research. 2014. Availablefrom:http://www.grandviewresearch.com/press-release/global-artificial-organ. 